\title{
Co-Design of Strain-Actuated Solar Arrays for Spacecraft Precision Pointing and Jitter Reduction
}

\author{
Christian M. Chilan, Daniel R. Herber ${ }^{\dagger}$ \\ University of Illinois at Urbana-Champaign, Urbana, IL, 61801, USA \\ Yashwanth Kumar Nakka \\ California Institute of Technology, Pasadena, CA, 91125, USA \\ James T. Allison \\ University of Illinois at Urbana-Champaign, Urbana, IL, 61801, USA \\ Jack B. Aldrich,! and Oscar S. Alvarez-Salazar** \\ Jet Propulsion Laboratory, California Institute of Technology, Pasadena, CA, 91109, USA
}

An earlier version of the material in this article was presented as paper 2016-0162 at the AIAA/ASCE/AHS/ASC Structures, Structural Dynamics, and Materials Conference, San Diego, CA, USA, 4-8 January 2016.

*Research Associate, Department of Aerospace Engineering, chilan@illinois.edu. AIAA Member

${ }^{\dagger}$ Graduate Student, Department of Industrial and Enterprise Systems Engineering, herber1@illinois.edu. AIAA Student Member

${ }^{\ddagger}$ Graduate Student, Department of Aerospace, ynakka@caltech.edu

$\S$ Associate Professor and Bren Scholar; Jet Propulsion Laboratory Research Scientist, Department of Aerospace, sjchung@caltech.edu. AIAA Senior Member

『Assistant Professor, Department of Industrial and Enterprise Systems Engineering, jtalliso@illinois.edu. AIAA Member

"Senior Member, Guidance and Control Analysis Group, Jack.B.Aldrich@jpl.nasa.gov. AIAA Member.

**Manager, Guidance and Control Analysis Group, oscar.s.alvarez-salazar@jpl.nasa.gov. AIAA Member.

1 of 43

American Institute of Aeronautics and Astronautics 
This work presents a novel spacecraft attitude control architecture using strain-actuated solar arrays (SASA) that does not require the use of conventional attitude control hardware. SASA enables attitude slewing maneuvers and precision-pointing (image acquisition) stares, while simultaneously

suppressing structural vibrations. Distributed piezoelectric actuators help achieve higher precision, higher bandwidth, and quieter operation than reaction wheels. To understand the design tradeoffs for this architecture, a framework for the integrated design of distributed structural geometry and distributed control is presented. The physical properties of the array are modeled and designed with respect to a piecewise linear distributed thickness profile. The distributed control is a voltage profile across the array modeled as a spatially continuous function. The dynamics of the system are modeled using a coupled ODE-PDE system using extended generalizations for hybrid coordinate systems. The combined physical- and control-system design, or co-design problem is investigated to understand the optimal performance of the system. Single-axis slew maneuvers of 7.2 milli-radians or 1485 arc seconds are achieved for a representative spacecraft model without increasing array mass or reducing array planform area. From additional tradeoff studies, a design criteria is revealed for the array structure and control strategy based on the optimal design tradeoff between large array inertia and fast structural dynamics. Moreover, the fundamental limits on SASA slew angle magnitude are demonstrated using an intuitive pseudorigid body dynamic model.

\section{Nomenclature}




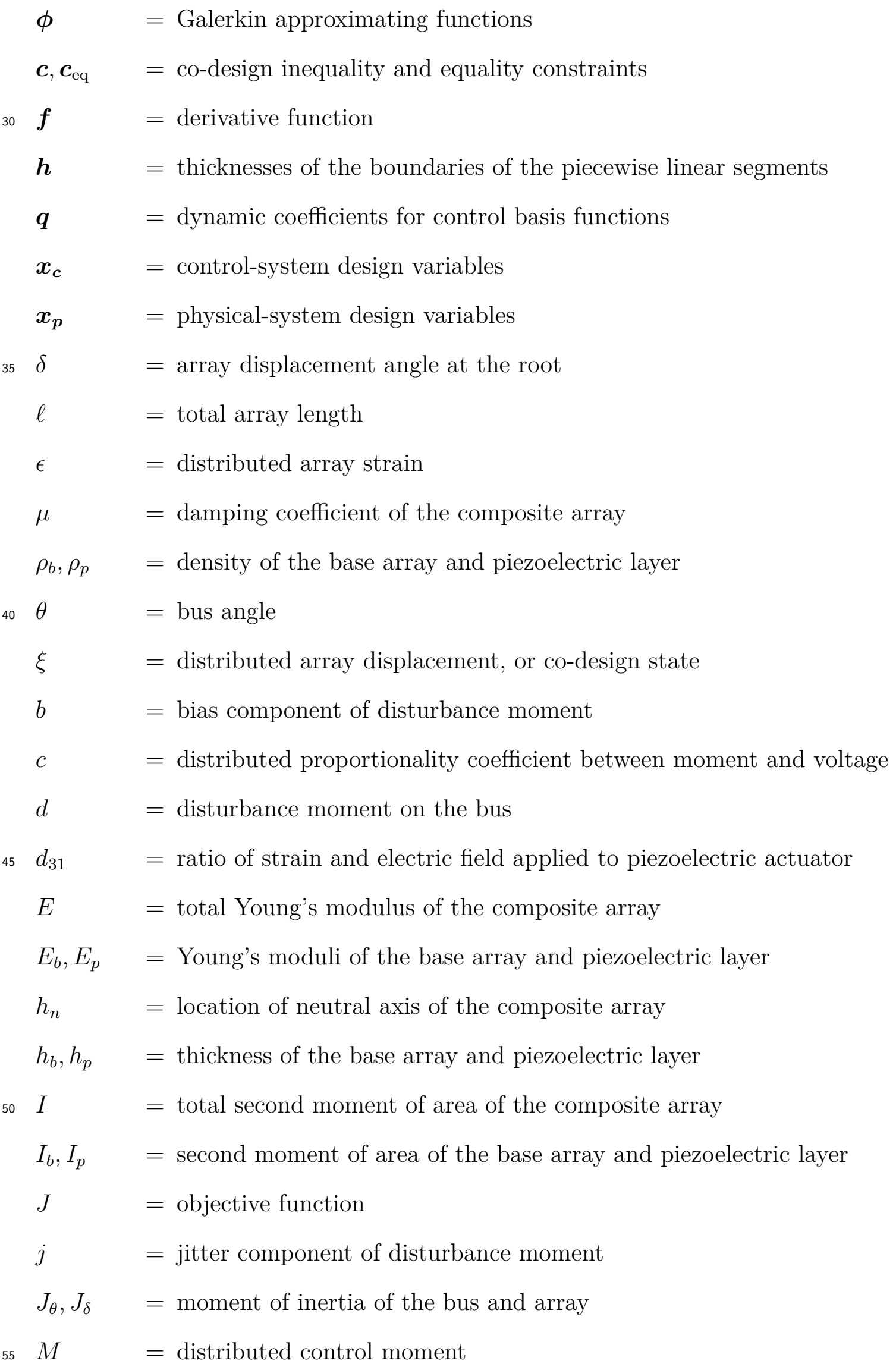




$\begin{aligned} m & =\text { mass of the composite array } \\ m_{R} & =\text { mass per unit length of the composite array } \\ m_{R b}, m_{R p} & =\text { mass per unit length of the base array and piezoelectric layer } \\ r & =\text { bus radius } \\ { }_{60} R_{\text {eff }} & =\text { effective inertia ratio of the bus-array system } \\ t & =\text { time variable } \\ t_{0}, \bar{t}, t_{f} & =\text { initial time, slewing time, and final time } \\ V & =\text { distributed control voltage } \\ w & =\text { array width } \\ { }_{65} x & \text { point location on the array from the root }\end{aligned}$

\section{Introduction}

Advancements in spacecraft technology accelerate discovery in Earth and space sciences; faster reorientation and ultra-quiet jitter-free operation for space observatories and optical links have the potential to transform the rate and quality of data obtained for scientific in- 
A unique capability of the proposed SASA pointing architecture is to perform attitude slewing maneuvers in addition to suppressing structural vibrations. Although the current bending limit of the arrays bounds the magnitude of the attitude maneuvers to the order of milli-radians or arc minutes, these advancements are important for high precision pointing, and when combined with other actuation technology for large reorientations may provide a feasible replacement for RWAs to improve pointing accuracy and reliability. Thus, the SASA control system is proposed as part of a hierarchical attitude control system where large-scale reorientations are carried out by magnetorquers or micro-thrusters. After the pointing target has been acquired, the SASA control system performs small-scale reorientations and pointing stability in the presence of jitter disturbances.

Strain-actuated solar arrays for precision pointing will require the arrays to behave more like a flexible structure than a rigid one. Space structures by necessity are extremely lightweight and flexible but vibrations from RWAs, reorientation maneuvers, and other disturbances degrade performance. These factors have given rise to the extensively-studied topic of Control-Structure Interaction (CSI). Most CSI literature has concentrated on reducing structural vibration through active damping,,$\underline{4}-\mathbf{z}$ i.e., using control systems to actively damp lower-frequency structural vibration modes without exciting higher-frequency modes. $\underline{\underline{8}} \underline{\underline{10}}$ This design paradigm has led to design rules, such as placing actuators according to locations of high strain for important structural modes. $\underline{\underline{8}} \underline{11}$ If the design objective is different from vibration reduction alone (e.g., more sophisticated motion control), then CSI rules may produce suboptimal results. As an illustrative example, consider Skylab where part of the control effort goes to reducing vibration and getting flexible bodies to behave as rigid bodies, leaving only the remaining control effort for productive tasks. ${ }^{9}$ Instead of using the proxy control objective of eliminating flexible behavior, a more elegant solution would be to improve overall system performance by working with elastic dynamics rather than fighting against them. In the integrated design and control study presented here we seek to utilize the flexible body dynamics to our advantage to provide new levels of system performance. Instead of using distributed actuation of solar arrays to damp out vibration as the primary 

a more comprehensive manner. $\underline{22}, \underline{23}$ For example, co-design has been used in the exploration 
of tradeoffs between plant redesign cost and system performance (energy consumption) in applications involving counterbalanced robotic manipulators. $\underline{24}$ Furthermore, it has been shown that co-design can yield solutions with significant performance improvements $\underline{25}, \underline{26}$ and at lower costs than CSI iterated sequential methods. $\underline{23}, \underline{27}, \underline{28}$ This type of methods typically performs optimization of the design vector at one level while performing system analysis, i.e. finding a feasible solution, in a second (lower) level. If the system analysis problem does not have a unique solution, the CSI iterated sequential method may converge to a suboptimal solution. $\frac{28}{}$ In contrast, nested co-design methods may define convex optimization problems in a second level for the control design of certain systems, e.g., dynamical systems that are linear in the states and the controls, that yield solutions that are not only feasible but also globally optimal. $\underline{23} \underline{\underline{29}}$

In most previous co-design studies, the physical aspects of the system design have been managed in a very simplified manner. For example, physical system (plant) design decisions have often been limited to actuator placement. $\underline{30}$, 31 Many co-design studies have used simplified plant models $\underline{9}, 17,24, \underline{32}$ that do not support exploration of changes to distributed geometric structural design, preventing full exploitation of the design synergy between structural tailoring and distributed control system design. A more ideal co-design method supports changes to distributed structural properties (e.g., changing structural shape affects how inertial and stiffness properties vary spatially). Structural tailoring coupled with control design has long been recognized as an important, yet formidable problem. $\underline{\underline{33}}$ Although there are examples of tailoring passive system dynamics to work optimally with active control using lumped plant stiffness, damping, and mass parameters as design variables, $\underline{\underline{ }}$ these methods cannot be extended to distributed parameter systems.

To summarize, much is known regarding the design of control systems and actuators for intelligent structures, but only if the structural design is held fixed. A few examples of fullyintegrated design exist, but only with simplified treatment of structural design. Since the proposed SASA pointing architecture involves inherent dynamic coupling between control actuation and flexible structural dynamics, it is a good case for a co-design study. In this 
work, distributed geometry — specifically, distributed array structure thickness - is optimized simultaneously with distributed moment control of the array structure.

An initial study of the SASA concept was performed previously, focusing on attitude control, to demonstrate its feasibility. It was shown that the spacecraft bus orientation can

The primary objectives of this work are to demonstrate the feasibility of the SASA attitude control architecture on a representative spacecraft system, to determine the optimal designs 
of the distributed array structure and controls, and to reveal qualitative design insights for intelligent structures with distributed geometric design. The contributions of this work are as follows:

1. We develop a coupled ODE-PDE model of the bus-array system based on extended generalization for hybrid coordinate systems. The elastic and inertial properties of the piezoelectric layer are included in the model.

2. We demonstrate bus attitude control using strain-actuated solar arrays with slewing maneuvers on the order of milli-radians or arc minutes for precision pointing and jitter reduction achieved in simulations.

3. We present a framework for the optimal integrated design of distributed structural geometry and distributed control systems.

4. We derive maximum bus slew limits achievable through SASA control using PRBDM analysis and co-design studies.

5. We present design guidelines, based on design optimization studies, for the distributed array structure and distributed control for the SASA system.

This paper is organized as follows. The models for the bus-array system, distributed composite array structure, distributed control, and PRBDM are presented in Sec. II. The formulation for the combined design of the distributed array structure and distributed control is presented in Sec. III. Analytical results based on PRBDM theory and numerical results of the co-design studies are discussed in Sec. IV. Results include the analysis of the optimal design tradeoff for the array structure, the optimal placement of segmented piezoelectric actuators, and parametric studies on passive damping and jitter disturbance. Finally, the conclusions are discussed in Sec. $\underline{\mathrm{V}}$.

9 of $\underline{43}$ 


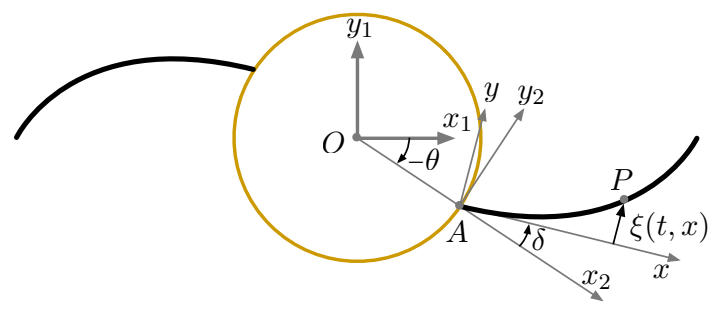

(a) Beam theory model.

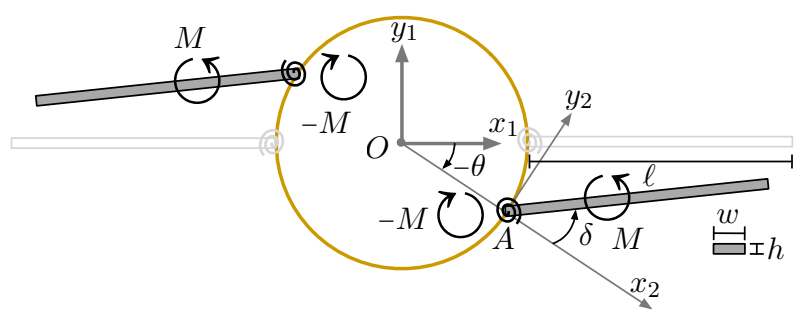

(b) Lumped parameter model.

Figure 1: Illustration of the two modeling approaches used to gain design insights.

\section{Modeling of the Strain-Actuated Solar Arrays and Rigid Spacecraft Bus}

Our modeling approach is based on the recent work on aircraft dynamics with flexible, articulated wings $\underline{\underline{43}}$ (see Ref. $\underline{\underline{44}}$ for details). The spacecraft motion is modeled as an Ordinary Differential Equation (ODE) of a simple cylinder, and the solar array structure is modeled as a PDE of a composite beam with thickness that can vary along its length.

\section{II.A. Partial Differential Equation (PDE) Model}

Here we assume that actuation is effected only through solar array strain actuators that produce strain at the solar array structure surface, resulting in array bending and a distributed moment due to strain actuator surface forces. The strain actuators do not interact with anything external to the spacecraft system, so the total system momentum must be conserved. Therefore, for a generally counter clockwise (CCW) movement of the solar array, the bus $(\theta)$ will rotate in the opposing CW direction allowing for attitude changes. This is evident in both the illustration of the beam theory coordinate system in Fig. 1a and its comparable PRBDM lumped parameter model in Fig. 1b.

\section{II.A.1. Coupled ODE-PDE Dynamic Model}

The coordinate systems used for the derivation of the Lagrangian of the system are shown in Fig. 1a. The model has two arrays with asymmetric actuation. Let the radius of the spacecraft body be $r$, and the spacecraft body rotation angle about origin $O$ be $\theta$. In 

model are discussed in Sec. II.A.3.

The moment applied on the array is $M(x, t)$ over the locations where a piezoelectric actuator is bonded; a small actuation gap $(0.5 \mathrm{~cm})$ was applied at the root and the tip to satisfy the boundary conditions. Using the explicit generalization for the hybrid coordinate systems approach, $\underline{\underline{4}}$ the equations of motion were derived. The dynamics in matrix form are:

$$
\int_{0}^{\ell}\left[\boldsymbol{M}_{s}\right]\left[\begin{array}{l}
\ddot{\theta} \\
\ddot{\xi}
\end{array}\right] d x+\int_{0}^{\ell}\left[\boldsymbol{C}_{s}\right]\left[\begin{array}{l}
\dot{\theta} \\
\dot{\xi}
\end{array}\right] d x+\left[\begin{array}{c}
0 \\
\int_{0}^{\ell}\left(2 E I \xi^{\prime \prime}+2 \mu E I \dot{\xi}^{\prime \prime}\right)^{\prime \prime} d x
\end{array}\right]=\left[\begin{array}{c}
d \\
\int_{0}^{\ell} 2 M^{\prime \prime} d x
\end{array}\right]
$$

where: $\quad\left[\boldsymbol{M}_{\boldsymbol{s}}\right]=\left[\begin{array}{cc}m_{11}(\xi) & m_{12} \\ m_{12} & m_{22}\end{array}\right]=\left[\begin{array}{cc}\left(J_{\theta} / \ell+2\left(m_{R}\left((x+r)^{2}+\xi^{2}\right)-p \xi^{\prime 2}\right)\right) & 2 m_{R}(x+r) \\ 2 m_{R}(x+r) & 2 m_{R}\end{array}\right]$,

$$
\left[\boldsymbol{C}_{s}\right]=\left[\begin{array}{cc}
c_{11}(\xi, \dot{\xi}) & c_{12}(\xi, \dot{\theta}) \\
-c_{12}(\xi, \dot{\theta}) & 0
\end{array}\right]=\left[\begin{array}{cc}
2 m_{R} \xi \dot{\xi}-2 p \xi^{\prime} \dot{\xi}^{\prime} & 2 m_{R} \xi \dot{\theta}+2\left(p \xi^{\prime}\right)^{\prime} \dot{\theta} \\
-2 m_{R} \dot{\theta}-2\left(p \xi^{\prime}\right)^{\prime} \dot{\theta} & 0
\end{array}\right],
$$

and where $(\cdot)^{\prime}=\frac{\partial}{\partial x}(\cdot)$. The axial tension in the array due to rotation is:

$$
A_{x}(\dot{\theta}, x)=\int_{r+x}^{r+\ell}\left[m_{R} \dot{\theta}^{2} s\right] d s=p(x) \dot{\theta}^{2} ; \text { where } p(x)=\int_{r+x}^{r+\ell} m_{R} s d s
$$

The term $\mu$ is used to model the structural damping in the solar array. A disturbance $d(t)$ acts on the bus as a torque input. The boundary conditions of the PDE in Eq. (1) are defined with respect to an axis fixed at the root of the solar array and radially outwards

11 of $\underline{43}$ 
from the center of the hub:

$$
\xi(0, t)=\xi^{\prime}(0, t)=0,\left.\quad E I\left(\xi^{\prime \prime}+\mu \dot{\xi}^{\prime \prime}\right)\right|_{x=\ell}=0,\left.\quad\left(E I\left(\xi^{\prime \prime}+\mu \dot{\xi}^{\prime \prime}\right)\right)^{\prime}\right|_{x=\ell}=0
$$

These boundary conditions specify zero deflection and slope of the deflected beam at the root, and no external force or moment at the free end.

The proposed SASA architecture is envisioned for high-precision pointing control. To maintain the structural integrity of the arrays and to take into account actuator limitations, bounds are defined for the array strain and control magnitude, which in turn limit array displacements to small values. Therefore, a linearized bus-array system can still predict the dynamics with sufficient accuracy as shown in Fig. $\underline{2}$. While the linear model does not approximate large array displacements accurately, displacements in our tests are small, and linearization makes the integrated structural and control optimization problem more tractable. This allows to conduct various parametric studies, which support the focus of this work on design methods and design insight. The following linearized equations are obtained from Eq. (1), and the boundary conditions remain the same:

$$
\begin{gathered}
\int_{0}^{\ell}\left[\boldsymbol{M}_{s l}\right]\left[\begin{array}{l}
\ddot{\theta} \\
\ddot{\xi}
\end{array}\right] d x+\left[\begin{array}{cc}
0 \\
\int_{0}^{\ell}\left(2 E I \xi^{\prime \prime}+2 \mu E I \dot{\xi}^{\prime \prime}\right)^{\prime \prime} d x
\end{array}\right]=\left[\begin{array}{c}
d \\
\int_{0}^{\ell} 2 M^{\prime \prime} d x
\end{array}\right] \\
\text { where: }\left[\boldsymbol{M}_{\boldsymbol{s l}}\right]=\left[\begin{array}{cc}
m_{11}(\xi) & m_{12} \\
m_{12} & m_{22}
\end{array}\right]=\left[\begin{array}{cc}
\left(J_{\theta} / \ell+2\left(m_{R}(x+r)^{2}\right)\right) & 2 m_{R}(x+r) \\
2 m_{R}(x+r) & 2 m_{R}
\end{array}\right]
\end{gathered}
$$

To demonstrate the validity of the linearization, open-loop simulations were performed for both the linear and nonlinear models. Two distinct tests were made for each model. On each test the system starts from rest. The first test then applies a step input of $200 \mathrm{~V}$ to the actuators. In the second test a sinusoidal input is used with a frequency of $1 \mathrm{~Hz}$ and an amplitude of $200 \mathrm{~V}$. The system parameters for these tests are given in Tables $\underline{1}$ and $\underline{2}$. The resulting steady state amplitudes of the bus angle for the nonlinear model are $4.6 \times 10^{-5}$ 
rad and $5.1 \times 10^{-5}$ rad for the step and sinusoidal inputs, respectively. The difference in bus angular rotation, $\left(\theta_{\text {nonlinear }}-\theta_{\text {linear }}\right)$, for these two tests is shown in Fig. $\underline{2}$ where $\theta_{\text {nonlinear }}$ and $\theta_{\text {linear }}$ are the open-loop bus angles obtained from nonlinear model and linear models, respectively. The error is extremely small, so the linear PDE model is used for the studies presented here.

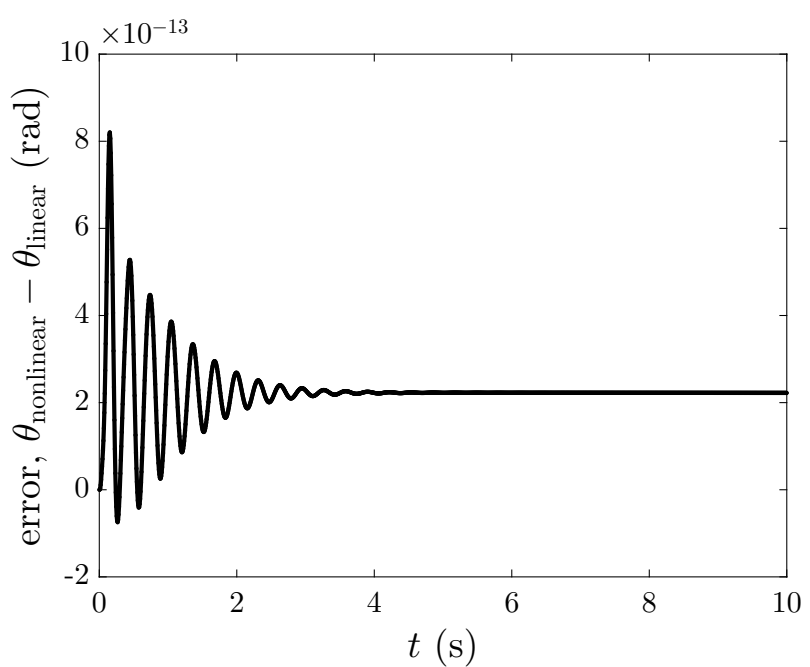

(a) Step input of $200 \mathrm{~V}$.

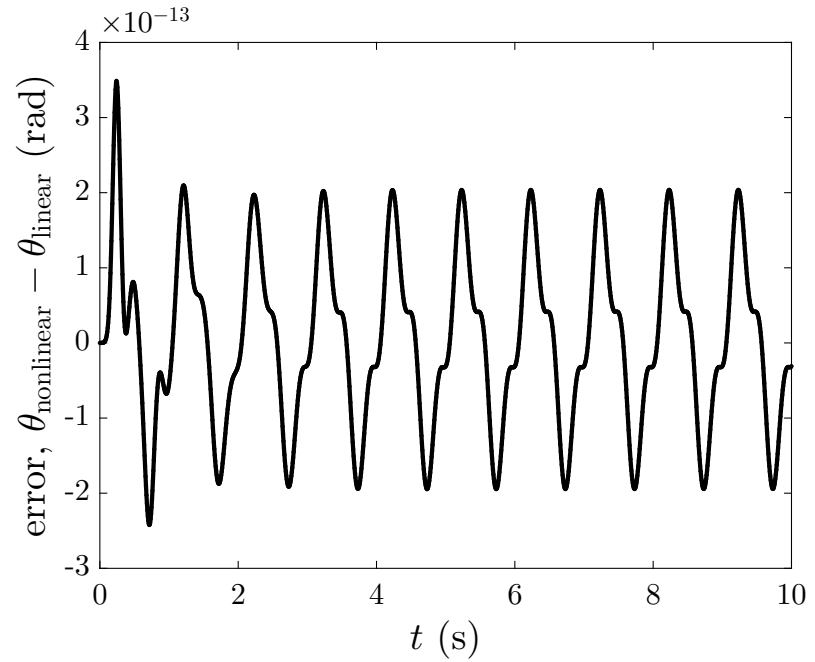

(b) Sinusoidal input of frequency of $1 \mathrm{~Hz}$ with amplitude of $200 \mathrm{~V}$.

Figure 2: Difference in bus angle trajectories between the linear and nonlinear models based on open-loop simulations with two different inputs.

\section{II.A.2. Galerkin Formulation}

To approximate numerically the PDE in Eqs. (1) and (3), we use a Galerkin formulation $\underline{40} \underline{41}, \underline{43}$ A linear combination of approximating functions is used to represent the array dynamic state $\frac{40}{}$ and distributed moment. These functions are chosen such that they satisfy the boundary conditions of the array, i.e., the fixed-free condition. The $j$ th approximating functions used for spatially distributed deflection and moment representations, respectively, are defined as: $\underline{\underline{40}}$

$$
\phi_{j}(x)=1-\cos \left(\frac{j \pi x}{\ell}\right)+\frac{1}{2}(-1)^{j+1}\left(\frac{j \pi x}{\ell}\right)^{2}, \quad \gamma_{j}(x)=\phi_{j}(x)+x^{j}+1
$$




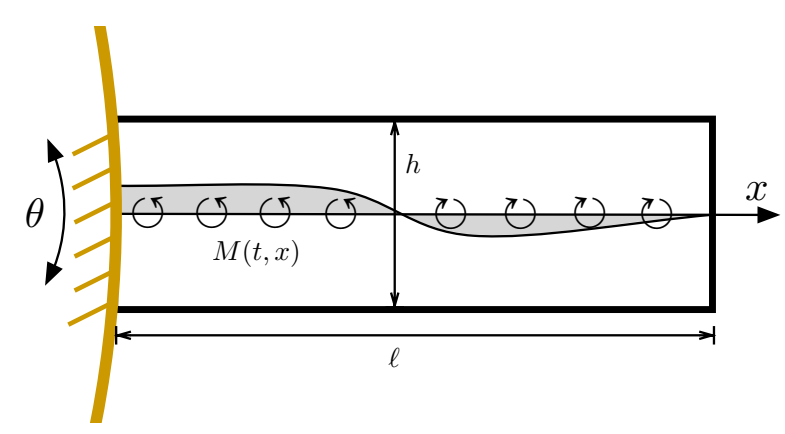

(a) Continuously distributed internal moment on a uniform thickness array.

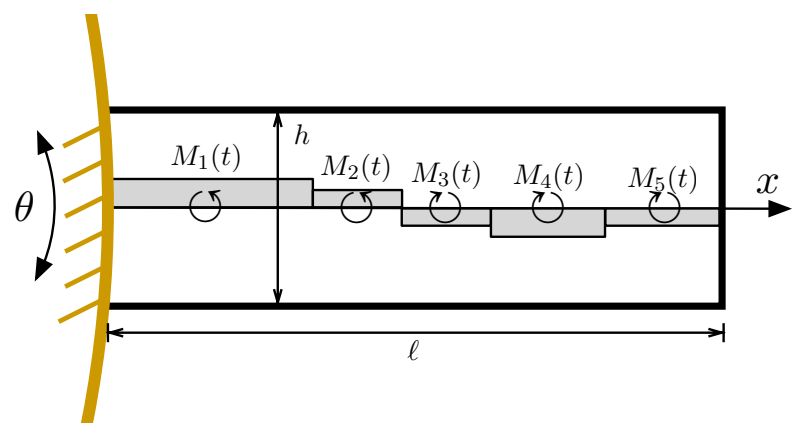

(b) Piecewise constant distributed internal moment on a uniform thickness array.

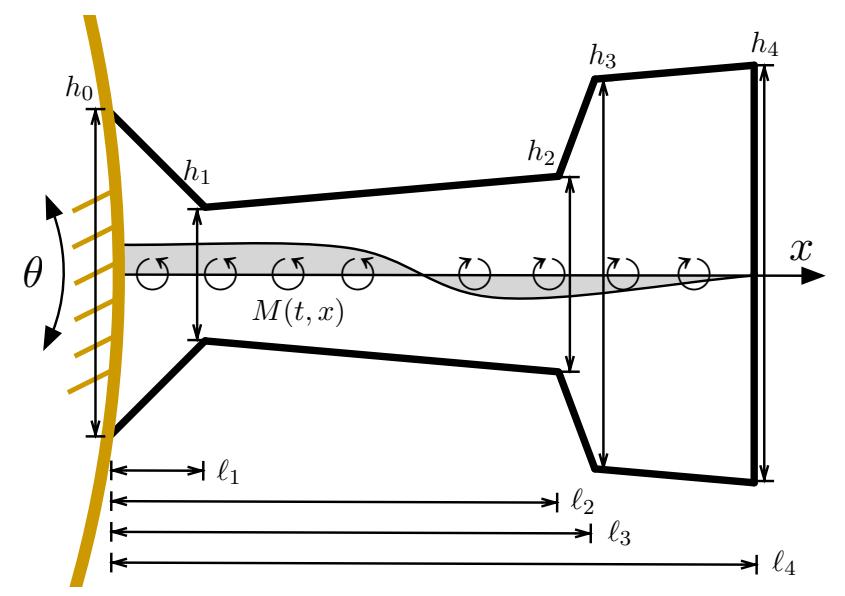

(c) Piecewise linear distributed array thickness.

Figure 3: Illustrations of various design representations for internally actuated array design problems for pointing.

The array deflection and distributed moment are then approximated as:

$$
\xi(x, t)=\boldsymbol{\phi}(x)^{T} \boldsymbol{\eta}(t), \quad M(x, t)=\boldsymbol{\gamma}(x)^{T} \boldsymbol{q}(t)
$$


A system of ODEs that approximate the PDE given above is derived $\underline{44}$ by minimizing weighted residual of the $\xi$ dynamics. Using the above formulation and defining additional matrices we obtain:

$$
\begin{aligned}
& {\left[\boldsymbol{M}_{\boldsymbol{g}}\right]\left[\begin{array}{l}
\ddot{\theta} \\
\ddot{\boldsymbol{\eta}}
\end{array}\right]+\left[\begin{array}{c}
0 \\
2[\boldsymbol{e}](\boldsymbol{\eta}+\mu \dot{\boldsymbol{\eta}})
\end{array}\right]=\left[\begin{array}{c}
d \\
\int_{0}^{\ell} 2 \boldsymbol{\phi} M^{\prime \prime} d x
\end{array}\right] } \\
& \text { where: }\left[\boldsymbol{M}_{\boldsymbol{g}}\right]=\left[\begin{array}{rr}
J_{\theta}+2 \int_{0}^{\ell} m_{R}(x+r)^{2} d x & 2[\boldsymbol{B}] \\
2[\boldsymbol{B}]^{T} & 2[\boldsymbol{A}]
\end{array}\right] \\
& {[\boldsymbol{A}]=\int_{0}^{\ell} m_{R} \boldsymbol{\phi} \phi^{T} d x, } {[\boldsymbol{B}]=\int_{0}^{\ell} m_{R}(x+r) \boldsymbol{\phi}^{T} d x, \quad[\boldsymbol{e}]=\int_{0}^{\ell} \boldsymbol{\phi}\left(E I \boldsymbol{\phi}^{\prime \prime T}\right)^{\prime \prime} d x }
\end{aligned}
$$

The boundary conditions with the approximating functions are then:

$$
\boldsymbol{\phi}(0)^{T}=\boldsymbol{\phi}^{\prime}(0)^{T}=0, \quad E I \boldsymbol{\phi}^{\prime \prime}(\ell)^{T}(\boldsymbol{\eta}+\mu \dot{\boldsymbol{\eta}})=0, \quad\left(E I \boldsymbol{\phi}^{\prime \prime T}\right)^{\prime}(\ell)(\boldsymbol{\eta}+\mu \dot{\boldsymbol{\eta}})=0
$$

\section{II.A.3. Structural Model of Composite Array}

The structural geometry of the array is also designed with the distributed moment. In this work, the length of the array and the distributed thickness are optimized. The spatially varying array thickness design is represented using piecewise linear segments. The length of the array is divided into multiple segments as shown in Fig. 3c. Segment lengths and slopes can be changed. The distributed array thickness design is parameterized using the absolute locations of the segment boundaries (quantified by the vector $\ell$ ), and the thickness at the segment boundaries (quantified by the vector $\boldsymbol{h}$ ). On the segment $j$, the thickness varies linearly with respect to $x$ as follows:

$$
h_{j}(x)=\left(h_{j+1}-h_{j}\right) \frac{x-\ell_{j}}{\ell_{j+1}-\ell_{j}}+h_{j}, \quad x \in\left[\ell_{j}, \ell_{j+1}\right]
$$

The array is laminated with a layer of piezoelectric material on the top surface which acts as an actuator and has the same width as the beam. It is also assumed that the entire 


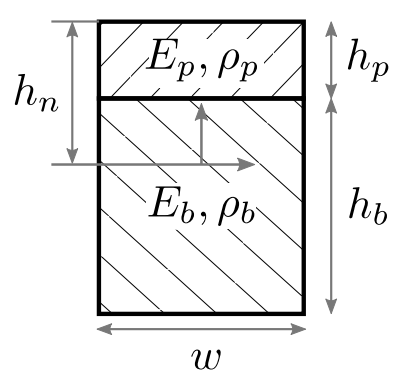

Figure 4: Cross section of the actuated array, modeled as a composite beam.

top surface of the array is covered with a piezoelectric layer of constant thickness $h_{p}$. The neutral axis of the composite beam is not at the center due to the inhomogeneous structural properties. Consider the cross section of the composite beam shown in Fig. $\underline{4}$. The distance from the neutral axis and the top surface of the array with piezoelectric material is $h_{n}$. The thickness of the base array and the piezoelectric layer are $h_{b}(x)$ and $h_{p}$, respectively. Note that $h_{b}(x)$ can vary spatially. The neutral axis location $h_{n}$, for each position $0 \leq x \leq \ell$, can be obtained by balancing the forces across the cross section and solving for $h_{n}$ :

$$
h_{n}=\frac{0.5 E_{p} h_{p}^{2}+E_{b} h_{b}\left(0.5 h_{b}+h_{p}\right)}{E_{p} h_{p}+E_{b} h_{b}}
$$

The area moments of inertia of the array, $I_{b}$, and the piezoelectric layer, $I_{p}$, about the neutral axis are:

$$
I_{b}=\frac{w h_{b}^{3}}{12}+w h_{b}\left(h_{p}+0.5 h_{b}-h_{n}\right)^{2}, \quad I_{p}=\frac{w h_{p}^{3}}{12}+w h_{p}\left(h_{n}-0.5 h_{p}\right)^{2}
$$

The total array rigidity is given by:

$$
E I=E_{b} I_{b}+E_{p} I_{p}
$$

The mass per unit length of the composite array is $m_{R}(x)=m_{R b}(x)+m_{R p}(x)$, where $m_{R b}(x)$ and $m_{R p}(x)$ are the mass per unit length of the base array and the piezoelectric material, respectively. The application of a voltage $V$ across the piezoelectric layer induces a moment $M$. This moment, due to only internal actuation, can be calculated by applying force balance 
across the cross section of the composite array: $\underline{\underline{4}}$

$$
\begin{aligned}
M(x, t) & =c(x) V(x, t), \\
\text { where: } c(x) & =\frac{d_{31} E_{b}^{2} w h_{b}^{3} E_{p} h_{b}\left(h_{b}+h_{p}\right)}{2\left(E_{b}^{2} h_{b}^{4}+4 E_{b} E_{p} h_{b}^{3} h_{p}+6 E_{b} E_{p} h_{b}^{2} h_{p}^{2}+4 E_{b} E_{p} h_{b} h_{p}^{3}+E_{p}^{2} h_{p}^{4}\right)},
\end{aligned}
$$

and $d_{31}$ is the ratio of the strain and the electric field applied across the piezoelectric layer when all the external forces are held constant. $\underline{45}$ Observe that the moment is proportional to the applied voltage.

\section{II.B. Pseudo-Rigid Body Dynamic Model}

A PRBDM was developed for the spacecraft system for the purpose of performing additional numerical studies that complement those based on the PDE model, including studies that yield qualitative insights that are difficult to obtain via the more sophisticated PDE model. The flexible solar arrays were modeled both with single and multi-link approximations. The single link model is presented here, where each array is modeled as a single rigid link connected to the spacecraft body via a revolute joint and a torsional spring (see Fig. 1b). This is a lumped compliance approximation of the distributed compliance of the actual solar array. However, these models only describe the behavior at a component level rather than the specific point-to-point variations, while Euler-Bernoulli beam in Sec. II.A does capture these variations.

Applying the Euler-Lagrange equation to the system in Fig. 1b we arrive at the following 
equations of motion:

$$
\begin{aligned}
& \mathcal{M}\left[\begin{array}{l}
\ddot{\theta} \\
\ddot{\delta}
\end{array}\right]+\mathcal{B}\left[\begin{array}{l}
\dot{\theta} \\
\dot{\delta}
\end{array}\right]+\mathcal{K}\left[\begin{array}{l}
\theta \\
\delta
\end{array}\right]=\boldsymbol{\tau} \\
& \text { where: } \mathcal{M}=\left[\begin{array}{cc}
J_{\theta}+2 J_{\delta}+2 m r^{2}+\frac{1}{2} m \ell^{2}+2 \ell m r \cos (\delta) & 2 J_{\delta}+m r \ell \cos (\delta)+\frac{1}{2} m \ell^{2} \\
2 J_{\delta}+m r \ell \cos (\delta)+\frac{1}{2} m \ell^{2} & 2 J_{\delta}+\frac{1}{2} m \ell^{2}
\end{array}\right] \\
& \mathcal{B}=\left[\begin{array}{cc}
-2 \ell m r \dot{\delta} \sin (\delta) & -\ell m r \dot{\delta} \sin (\delta) \\
\ell m r \dot{\theta} \sin (\delta) & 2 b
\end{array}\right], \quad \mathcal{K}=\left[\begin{array}{cc}
0 & 0 \\
0 & 2 k
\end{array}\right], \quad \boldsymbol{\tau}=\left[\begin{array}{c}
q \\
2 M
\end{array}\right] \\
& m=\rho \ell w h, \quad J_{\delta}=\frac{1}{12} m\left(\ell^{2}+h^{2}\right),
\end{aligned}
$$

and where $k$ is the torsional spring stiffness and $b$ is the damping constant at the revolute joints. Solving the eigenvalue problem $\left(\mathcal{M}^{-1} \mathcal{K}=\boldsymbol{\omega}^{2}\right)$ gives the natural frequencies of the system:

$$
\begin{aligned}
& \omega_{1}^{2}=0 \\
& \omega_{2}^{2}=k \frac{2 m \ell^{2}+8 m \ell r \cos (\delta)+8 m r^{2}+4 J_{\theta}+8 J_{\delta}}{-2 \ell^{2} m^{2} r^{2} \cos (\delta)^{2}+2 \ell^{2} m^{2} r^{2}+J_{\theta} \ell^{2} m+8 J_{\delta} m r^{2}+4 J_{\theta} J_{\delta}}=\frac{k}{J_{\text {eff }}(\delta, \ell, h, w)}
\end{aligned}
$$

One of the eigenfrequencies is zero since the system permits a rigid body mode. The two mode shapes are:

$$
\boldsymbol{\Psi}=\left[\begin{array}{ll}
\boldsymbol{\psi}_{1} & \boldsymbol{\psi}_{2}
\end{array}\right]=\left[\begin{array}{cc}
1 & -\frac{m \ell^{2}+2 m r \ell \cos (\delta)+4 J_{\delta}}{m \ell^{2}+4 m r \ell \cos (\delta)+4 m r^{2}+2 J_{\theta}+4 J_{\delta}} \\
0 & 1
\end{array}\right]
$$

We note that the nonrigid mode eigenfrequency $\left(\omega_{2}\right)$ and eigenvector $\left(\boldsymbol{\psi}_{2}\right)$ are not constant but depend on the path of the array. The total angular momentum of the system is:

$$
\chi=\left(\frac{1}{2} \ell^{2} m+2 \ell m r \cos (\delta)+2 m r^{2}+J_{\theta}+2 J_{\delta}\right) \dot{\theta}+\left(\frac{1}{2} \ell^{2} m+\ell m r \cos (\delta)+2 J_{\delta}\right) \dot{\delta}
$$

Since internal moments cannot change the total angular momentum of the system, the only

18 of $\underline{43}$ 
mode that is present in the absence of external moments is the momentum conserving mode $\psi_{2}$

\section{Co-Design Problem Formulation}

The objective of the co-design study is to provide insights into how the actively-controlled solar array should be designed to optimize the performance in terms of attitude slewing and jitter reduction. A balanced co-design approach is utilized where physical-system (geometric specification of the solar array) and control-system (open-loop voltage trajectories) design are considered in an equally comprehensive manner. $\underline{23}$ A general simultaneous co-design formulation (with a fixed time horizon) is:

$$
\begin{array}{rl}
\min _{\boldsymbol{x}_{c}, \boldsymbol{x}_{p}} & J\left(t, \boldsymbol{\xi}, \boldsymbol{x}_{c}, \boldsymbol{x}_{p}\right) \\
\text { subject to: } & \dot{\boldsymbol{\xi}}(t)-\boldsymbol{f}\left(t, \boldsymbol{\xi}, \boldsymbol{x}_{c}, \boldsymbol{x}_{p}\right)=\mathbf{0} \\
& \boldsymbol{c}\left(t, \boldsymbol{\xi}, \boldsymbol{x}_{c}, \boldsymbol{x}_{p}\right) \leq \mathbf{0} \\
& \boldsymbol{c}_{\mathrm{eq}}\left(t, \boldsymbol{\xi}, \boldsymbol{x}_{c}, \boldsymbol{x}_{p}\right)=\mathbf{0}
\end{array}
$$

where $\boldsymbol{x}_{c}$ are the control design variables, $\boldsymbol{x}_{p}$ are the physical system design variables, and $\boldsymbol{\xi}$ are the states in Eq. (7), Eq. (18a) is a general performance objective, Eq. (18b) enforces the feasible dynamics of a set of first-order ordinary differential equations (ODE), Eq. (18c) are general inequality constraints, and Eq. (18d) are general equality constraints. Here the physical system design is parameterized by $\boldsymbol{h}$ and $\boldsymbol{\ell}$ with eight distinct linear segments. The control system design variable is defined here as $\boldsymbol{x}_{c}:=\boldsymbol{q}(t)$; these control trajectories are used to compute $M(x, t)$ and $V(x, t)$.

The spacecraft control task is divided into two consecutive phases; $\bar{t}$ is the time duration of the first phase. The first phase (slewing) concentrates on rotating the bus from an initial angular displacement $\theta\left(t_{0}\right)$ back to $\theta=0$ at time $\bar{t}$. In the second phase (pointing) the bus is held inertially fixed for precision pointing. Any vibrations generated during slewing must be 
Table 1: Bus parameters.

\begin{tabular}{rl}
\hline \hline$J_{\theta}$ & $372.49 \mathrm{~kg} \mathrm{~m}^{2}$ \\
$r$ & $1.02 \mathrm{~m}$ \\
\hline \hline
\end{tabular}

Table 2: Array and piezoelectric material parameters.

\begin{tabular}{cc|cl|cl}
\hline \hline$\ell_{\text {nominal }}$ & $1.575 \mathrm{~m}$ & $E_{b}$ & $1.57 \mathrm{GPa}$ & $E_{p}$ & $62 \mathrm{GPa}$ \\
$h_{\text {nominal }}$ & $0.018 \mathrm{~m}$ & $\rho_{b}$ & $332.03 \mathrm{~kg} / \mathrm{m}^{3}$ & $\rho_{p}$ & $7800 \mathrm{~kg} / \mathrm{m}^{3}$ \\
$w_{\text {nominal }}$ & $1.862 \mathrm{~m}$ & $\mu$ & $10^{-4} \mathrm{~s}$ & $h_{p}$ & $2 \times 10^{-4} \mathrm{~m}$ \\
\hline \hline
\end{tabular}

damped out during the pointing phase. The objective function is to maximize the slewing angle, demonstrating the maximum capability of the SASA attitude control system:

$$
J=-\theta\left(t_{0}\right)
$$

where $t \in\left[t_{0}, t_{f}\right]=[0 \mathrm{sec}, \bar{t}+1 \mathrm{sec}]$ and $\bar{t}$ is solved at various values between $0.12 \mathrm{~s}$ and $4 \mathrm{~s}$. This parametric sweep on $\bar{t}$ helps quantify the tradeoff between the competing objectives of slew angle maximization and slew time minimization. The dynamic constraint uses the linear ODE defined in Eq. (7) with 4 approximating functions. The physical parameters for the bus, array, and piezoelectric layer are shown in Tables $\underline{1}$ and $\underline{2}$. A disturbance moment $d(t)$ on the bus is present during the slewing and pointing phases. It consists of a jitter component $j(t)$ (e.g., vibrations similar to those that arise from moving parts such as pumps) and a bias component $b$ (e.g., due to solar radiation pressure, atmospheric drag):

$$
d(t)=b+j(t)=10^{-4} \mathrm{Nm}+2 \times 10^{-3} \sin (50 t) \mathrm{Nm}
$$

The initial configuration is stationary with an initial bus orientation $\theta_{0}=\theta\left(t_{0}\right) \neq 0$. The initial states are:

$$
\boldsymbol{\xi}_{t=t_{0}}^{\top}=\left[\begin{array}{llll}
\theta & \dot{\theta} & \boldsymbol{\eta}^{\top} & \dot{\boldsymbol{\eta}}^{\top}
\end{array}\right]_{t=t_{0}}^{\top}=\left[\begin{array}{llll}
\theta_{0} & 0 & \mathbf{0}^{\top} & \mathbf{0}^{\top}
\end{array}\right]^{\top}
$$


The bus angle and angular rate are constrained to 0 during the pointing phase. This simultaneously meets the pointing task and eliminates jitter if a feasible solution is found:

$$
\begin{array}{ll}
|\theta(t)|=0, & t \in\left[\bar{t}, t_{f}\right] \\
|\dot{\theta}(t)|=0, & t \in\left[\bar{t}, t_{f}\right]
\end{array}
$$

Numerical experiments indicate these constraints can be satisfied in all but very unusual cases. PEMA actuation magnitude is constrained to satisfy maximum voltage restrictions:

$$
|V(x, t)|=\left|\frac{M(x, t)}{c(x)}\right| \leq 300 \mathrm{~V}
$$

The array surface strain is constrained to be less than $0.1 \%$ to maintain structural integrity:

$$
|\epsilon(x, t)|=\left|\left(h_{b}(x)+h_{p}-h_{n}(x)\right) \xi^{\prime \prime}(t)\right| \leq 10^{-3}
$$

Traditional silicon-based solar cells can withstand strain levels on the order of $0.1 \%$. $\underline{\text { t }}$ However, recent advances allow the manufacturing of flexible solar cells that can achieve strain levels on the order of $10 \%, \underline{47}$ as well as fiber-shaped solar cells that can be woven into textiles. $\underline{48}$

The total array length $\ell_{n} \equiv \ell$, linear array segment lengths, and array thickness values have manufacturing and operational constraints:

$$
\begin{gathered}
0.5 \mathrm{~m} \leq \ell_{n} \leq 2.5 \mathrm{~m} \\
0.05 \mathrm{~m} \leq \ell_{i+1}-\ell_{i} \leq 1 \mathrm{~m} \quad i=0,1, \ldots, n \\
0.009 \mathrm{~m} \leq h_{i} \leq 0.055 \mathrm{~m}
\end{gathered}
$$

The array volume is constrained to be less than the nominal value in order to avoid increasing the payload mass and delivery costs. This is proportional to the array structure cross- 
sectional area:

$$
\sum_{i=1}^{n} \frac{w}{2}\left(h_{i-1}+h_{i}\right)\left(\ell_{i}-\ell_{i-1}\right) \leq 0.054 \mathrm{~m}^{3}
$$

Array planform area is constrained to the nominal value to maintain the same level of power generation:

$$
\ell_{n} w=2.932 \mathrm{~m}^{2}
$$

Direct orientation of the solar arrays towards the sun requires the attitude rotation axis to be normal to the sun-spacecraft vector. Solar power generation is a function of array area and orientation (and other factors). The planform area constraint is intended to be large enough to ensure adequate overall power generation even when arrays are not oriented directly toward the sun. A more sophisticated and accurate approach would model power generation directly across a range of maneuvers and insolation conditions. An investigation of the tradeoff between power generation and attitude control is a topic for future work.

This completes the exposition of the co-design problem formulation; objective and con-

straint functions are summarized in Table 3. Note that some optimization variables (states and controls) are linear while other variables (plant parameters) are nonlinear in the codesign formulation. A traditional approach to solve this type of problems is to use nested co-design. $\underline{23}, \underline{29}, \underline{49}$ This approach consists of an outer-loop that finds the optimal plant parameters, while the inner-loop finds the optimal states and controls histories for each point in the plant parameter space sampled by the outer-loop. In this way, the inner-loop can return the cost of a particular plant design to the outer-loop. Since the the optimal control problem in the inner-loop is linear, it can be formulated and solved as a linear program (LP) using a direct transcription method. $\underline{29}, \underline{50} \underline{52}$ Direct transcription has been used traditionally in trajectory optimization. $\underline{51}, \underline{53}, \underline{54}$ A custom transcription code was used to transform the infinite dimensional optimal control problem into a finite dimensional optimization problem.

A feasible solution for the inner-loop problem is globally optimal because it is formulated 
as an LP program. However, the nonlinear dependencies on the plant parameters in the outer-loop require special attention to ensure that the global optimum is found. The outer loop is implemented using MATLAB patternsearch, while the inner-loop is implemented (Latin hypercube sampling) and polling options to help find the global solution.

The results for a number of minor variations of this formulation will be discussed next after a short study on the fundamental limits of a slewing maneuver with SASA utilizing a reduced form of this co-design formulation and the PRBDM model.

Table 3: Summary of co-design problem formulation.

\begin{tabular}{|c|c|c|c|c|c|c|}
\hline \multirow[b]{2}{*}{ Name } & \multirow[b]{2}{*}{ Eq. \# } & \multicolumn{5}{|c|}{ Linear w.r.t. } \\
\hline & & Type & $\xi$ & $x_{c}$ & $x_{p}$ & Level \\
\hline Max. Slew Amount & Eq. $(19 a)$ & Eq. (18a) & Yes & & & Both \\
\hline Dynamics & Eq. (7) \& Eq. (19b) & Eq. $(\overline{18 b})$ & Yes & Yes & No & Inner \\
\hline Initial Conditions & $\overline{\mathrm{Eq}} \cdot(19 \mathrm{c})$ & Eq. $(\overline{18 d})$ & Yes & & & Inner \\
\hline Pointing & Eq. (19d) \& Eq. (19e) & Eq. $(\overline{18 d})$ & Yes & & & Inner \\
\hline Voltage Limits & $\overline{\mathrm{Eq}} \cdot(19 f)$ & Eq. $(\overline{18 c})$ & & Yes & No & Inner \\
\hline Strain Limits & Eq. $(\overline{19 g})$ & Eq. $(\overline{18 c})$ & Yes & & No & Inner \\
\hline Geometry Bounds & Eq. $(\overline{19 h})$ & Eq. $(\overline{18 c})$ & & & Yes & Outer \\
\hline Array Volume & Eq. $\overline{(19 \mathrm{i})}$ & Eq. $(\overline{18 \mathrm{c}})$ & & & No & Outer \\
\hline Planform Area & Eq. $(\overline{19 j})$ & Eq. $(\overline{18 d})$ & & & No & Outer \\
\hline
\end{tabular}

\section{Analytical and Numerical Results for SASA System}

\section{IV.A. Maximum Slewing Bounds using the PRBDM}

The momentum of the PRBDM system is given in Eq. (17), and it must be conserved if no external disturbance acts on the spacecraft $(d \equiv 0)$. Assuming zero initial momentum, we 
can integrate the momentum equation to determine the relationship between $\theta$ and $\delta$ :

$$
\begin{gathered}
0=\left(\frac{1}{2} \ell^{2} m+2 \ell m r \cos (\delta)+2 m r^{2}+I_{\theta}+2 I_{\delta}\right) \dot{\theta}+\left(\frac{1}{2} \ell^{2} m+\ell m r \cos (\delta)+2 I_{\delta}\right) \dot{\delta} \\
:=I_{1}(\ell, h, w, \delta) \dot{\theta}+I_{2}(\ell, h, w, \delta) \dot{\delta} \\
\dot{\theta}=-\frac{I_{2}(\delta, \cdot)}{I_{1}(\delta, \cdot)} \dot{\delta} \\
\theta\left(t_{f}\right)-\theta\left(t_{0}\right)=-\int_{t_{0}}^{t_{f}} \frac{I_{2}(\delta, \cdot)}{I_{1}(\delta, \cdot)} \dot{\delta} d t
\end{gathered}
$$

The question we are trying to answer requires an upper bound on $\left|\theta\left(t_{f}\right)-\theta\left(t_{0}\right)\right|$. We can find a reasonable upper bound by determining the maximum value of the integrals. Recall that $m$ and $I_{\delta}$ are dependent on the geometric physical design variables. Since the geometric variables are positive and it is reasonable to assume $\cos (\delta)>0$ (panel angle must be smaller than $|\delta|<\pi / 2$ ), then we see that the following difference is strictly positive: $I_{1}-I_{2}=$ $\ell m r \cos (\delta)+2 m r^{2}+I_{\theta}>0$. Therefore, the effective inertia ratio, $R_{\text {eff }}:=I_{2} / I_{1}$, must be between 0 and 1.

If $|\delta(t)| \leq \delta_{\max }$ is small, then $R_{\text {eff }}$ is nearly time-independent. Observe also that the effective inertia ratio is maximized when $\delta=0$. Therefore, we will use this maximal value, denoted $R_{\text {eff,max }}$, as a time-independent value to arrive at the following inequality:

$$
\begin{aligned}
\left|\theta\left(t_{f}\right)-\theta\left(t_{0}\right)\right| & \leq R_{\text {eff,max }}\left|-\int_{t_{0}}^{t_{f}} \dot{\delta} d t\right| \\
& \leq R_{\text {eff, } \max }\left|\delta\left(t_{0}\right)-\delta\left(t_{f}\right)\right|
\end{aligned}
$$

Assuming $\delta\left(t_{0}\right)=0, \theta\left(t_{f}\right)=0$, and that the prescribed bound on $\delta$ is hit at $t_{f}$, then we have:

$$
\left|\theta_{0}\right| \leq R_{\text {eff,max }} \delta_{\max } \leq \delta_{\max }
$$

Therefore we expect the maximum change for the bus angle to be bounded above by the allowable change in panel angle using only internal actuation of the solar array. This implies that if only milli-radian deflections of the array are feasible, we can only achieve, at most, 
milli-radian changes in bus orientation. Additional novel solutions for SASA such as revolute joints that allow for large changes in the effective inertia ratio without violating conservation of momentum may extend this limit.

A comparable $\delta_{\max }$ condition for a continuous array is a strain bound. If we want the largest value for $\delta$, analogously the strain will be at its maximum possible value at each point on the arrays. Using the constant thickness solar array in Fig. 1b, we can calculate this bound as $\delta_{\max }=(\ell / h) \epsilon_{\max }$. With this relationship, we can calculate the predicted maximum slewing bound. Two cases are shown in Table 4: nominal geometry and maximum allowable value for $\ell$. The latter case achieves the maximum value of the slew bound since both $R_{\text {eff,max }}$ and $\delta_{\max }$ are maximized. We can also determine the actual maximum slew bound using the optimal control problem in Sec. III (i.e., fixed geometry) without the voltage constraint. This result is also shown in Table $\underline{4}$ and indeed the bound is verified. The bound is not tight because the optimal voltage trajectories did not strain all of the array to the prescribed bound but only most of the array; thus, this assumption was only partially valid. In the next section, the study will consider the other design constraints and allow piecewise-linear array thickness changes. The bounds in this section do not consider all the design constraints and therefore are only approximate indications of the maximum possible slewing performance.

Table 4: Summary of results for maximum slewing bounds using the PRBDM.

\begin{tabular}{rccc}
\hline \hline Study & $R_{\text {eff,max }}$ & $R_{\text {eff, } \max } \delta_{\max }$ & Actual \\
\hline Nominal Geometry & 0.12 & $0.0108 \mathrm{rad}\left(0.62^{\circ}\right)$ & $>0.0063 \mathrm{rad}\left(0.36^{\circ}\right)$ \\
Maximal $\ell$ & 0.22 & $0.0301 \mathrm{rad}\left(1.73^{\circ}\right)$ & $>0.0176 \mathrm{rad}\left(1.01^{\circ}\right)$ \\
\hline \hline
\end{tabular}

\section{IV.B. Maximum Slewing Bounds using the Co-design Problem Formulation}

In this section we study three variations of geometric design representation in the co-design problem introduced in Sec. III. These are denoted nominal geometry (NG), variable length (VL), and piecewise linear segments (PLS). The geometric constraints for each case are summarized in Table $\underline{5}$. The NG case does not involve physical-system design since it is fixed and is only included in this study as a performance baseline. For the VL case, $\ell$ is the 


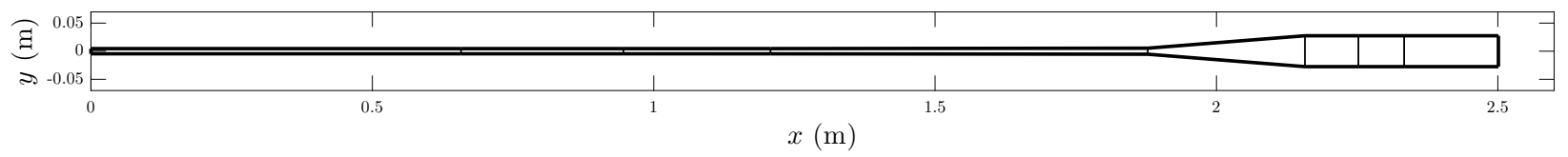

(a) Array design with maximal array inertia (drawn to scale).

Not Drawn to Scale, $y$ axis $\approx 25 \times$ magnification

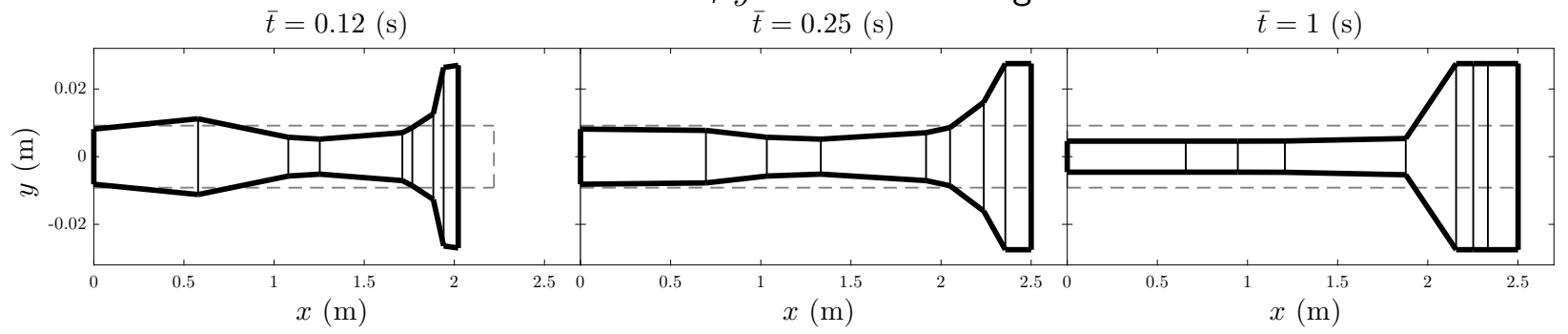

(b) Array designs for both Variable Length (dashed) and Piecewise Linear Segments (solid) studies.

Figure 5: Optimal array designs.

Table 5: Geometric constraints for the co-design problem variations.

\begin{tabular}{rccc}
\hline \hline Variation & Length $(\mathrm{m})$ & $\begin{array}{c}\text { Array Property } \\
\text { Thickness }(\mathrm{m})\end{array}$ & Volume $\left(\mathrm{m}^{3}\right)$ \\
\hline NG & 1.575 & 0.018 & 0.054 \\
VL & $0.5 \leq \ell \leq 2.5$ & 0.018 & 0.054 \\
PLS & $0.5 \leq \ell \leq 2.5$ & $0.009 \leq h(x) \leq 0.055$ & $\leq 0.054$ \\
\hline \hline
\end{tabular}

The maximum slewing results for each of the three variations are summarized in Fig. $\underline{6}$. As expected, the NG case achieved the smallest maximum slewing angle (0.9 mrad) for all the tested slewing times. This result indicates that the performance level desired may not be 


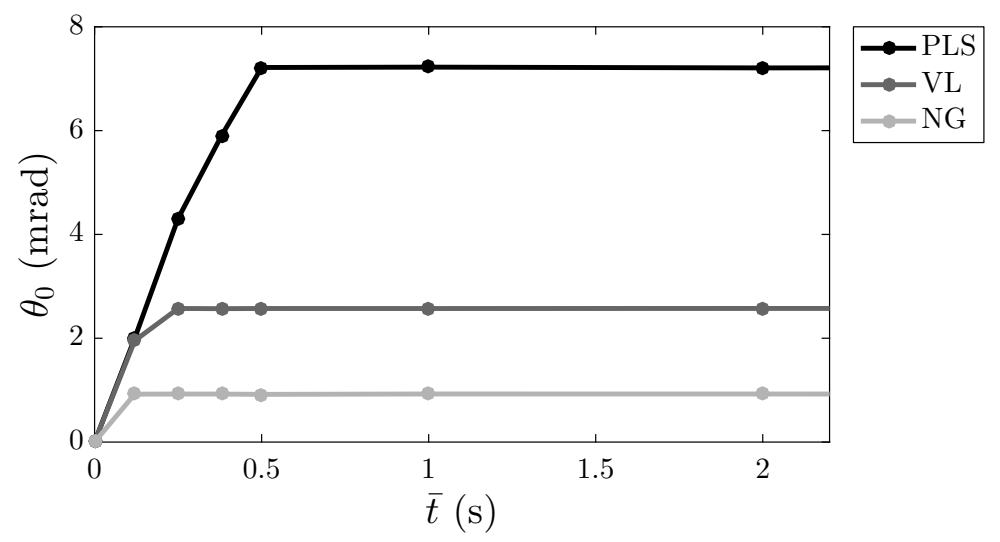

Figure 6: Parametric study of maximum slewing angle with respect to slewing time.

achievable through control design alone. Furthermore, the peak maximum achievable slewing angles for the VL and PLS cases (2.6 mrad and $7.2 \mathrm{mrad}$, respectively) are consistent with the results of Sec. IV.A. The optimal array designs are shown in Fig. 5b. We observed that the optimal PLS geometries for slewing times $\geq 0.5 \mathrm{~s}$ were similar. In addition, optimal VL designs for slewing times $\geq 0.25 \mathrm{~s}$ are equal. The optimal trajectories for the bus angle and angular rate for the PLS and VL cases are shown in Fig. 7. These figures show that, through only internal actuation of the solar arrays, the slew maneuvers were performed and then the bus was held fixed (i.e., $\theta \equiv 0$ and $\dot{\theta}=0$ ) for $1.0 \mathrm{sec}$ all while managing jitter.

Note that for the VL case with slewing times of $0.25 \mathrm{sec}$ and $1.0 \mathrm{sec}$, the bus angle trajectories are different, but the same slewing angle is achieved. Additional control-designonly problem formulations were conducted with $\bar{t}$ up to $30 \mathrm{~s}$ and with the array design fixed as the optimal design from the $4 \mathrm{~s}$ slew time PLS solution. It was found that the maximum slewing angle remained equal to $7.2 \mathrm{mrad}$, indicating that a fundamental limit was reached. The limiting factor preventing larger slew angles here is the actuator voltage limit, as opposed to momentum limitations as detailed in Sec. IV.A. Figure $\underline{8}$ illustrates that the actuator voltage is saturated during the pointing phase.

The array displacements for the PLS and VL cases are shown in Fig. 9. Results from slewing and pointing phases are shown separately. Conservation of angular momentum with negligible material damping in the bus-array system provides a natural explanation of these numerical results. For example, rotating the bus in the clockwise direction requires the array 

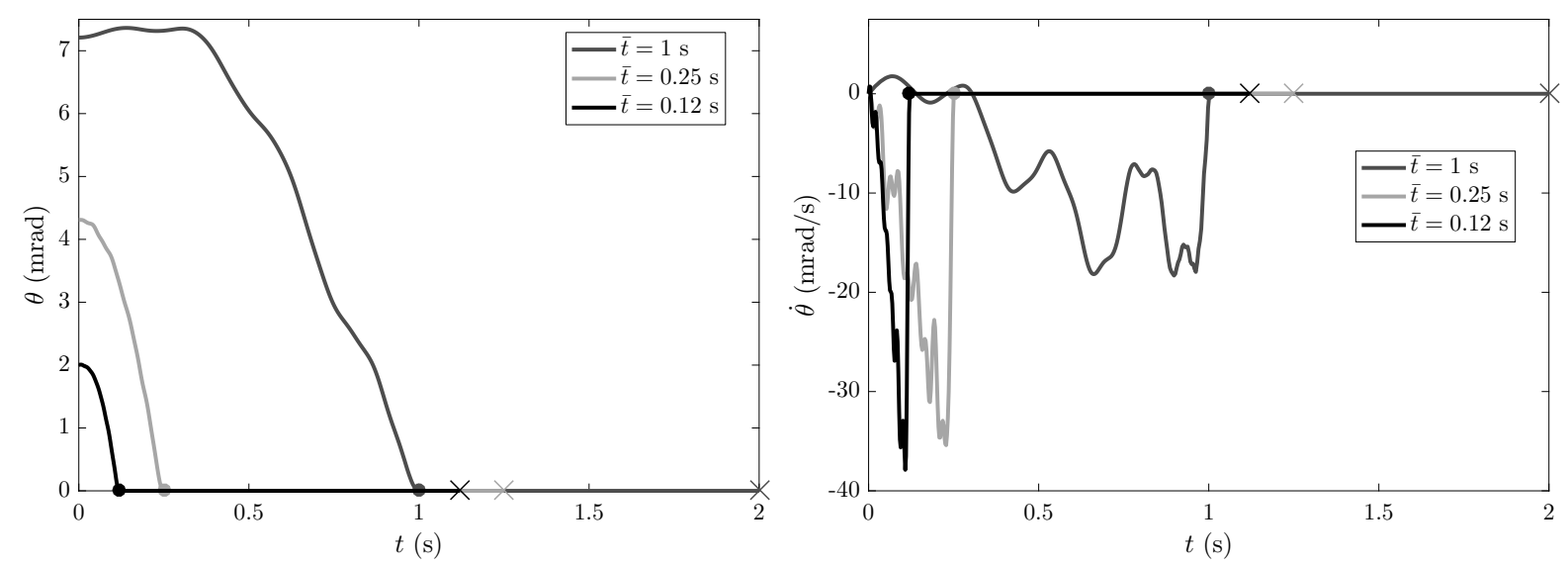

(a) Piecewise Linear Segments (PLS).
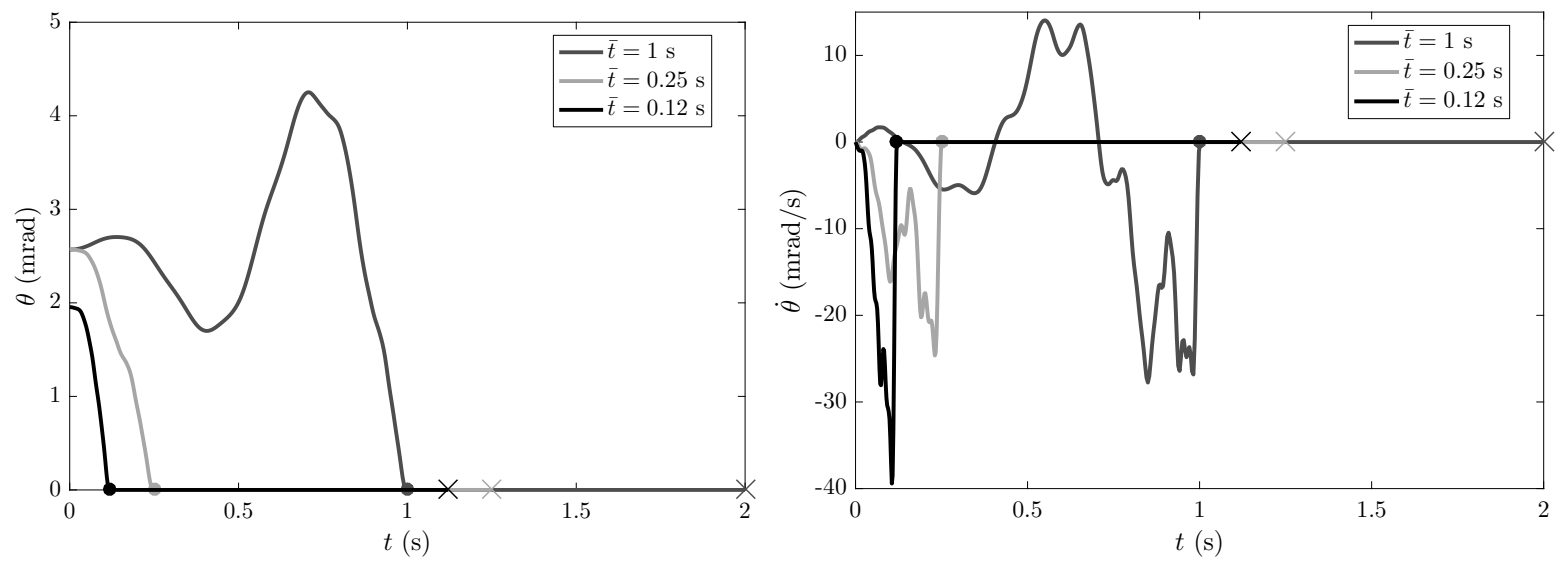

(b) Variable Length (VL).

Figure 7: Bus angle and angular rate trajectories for select values of $\bar{t}$. 

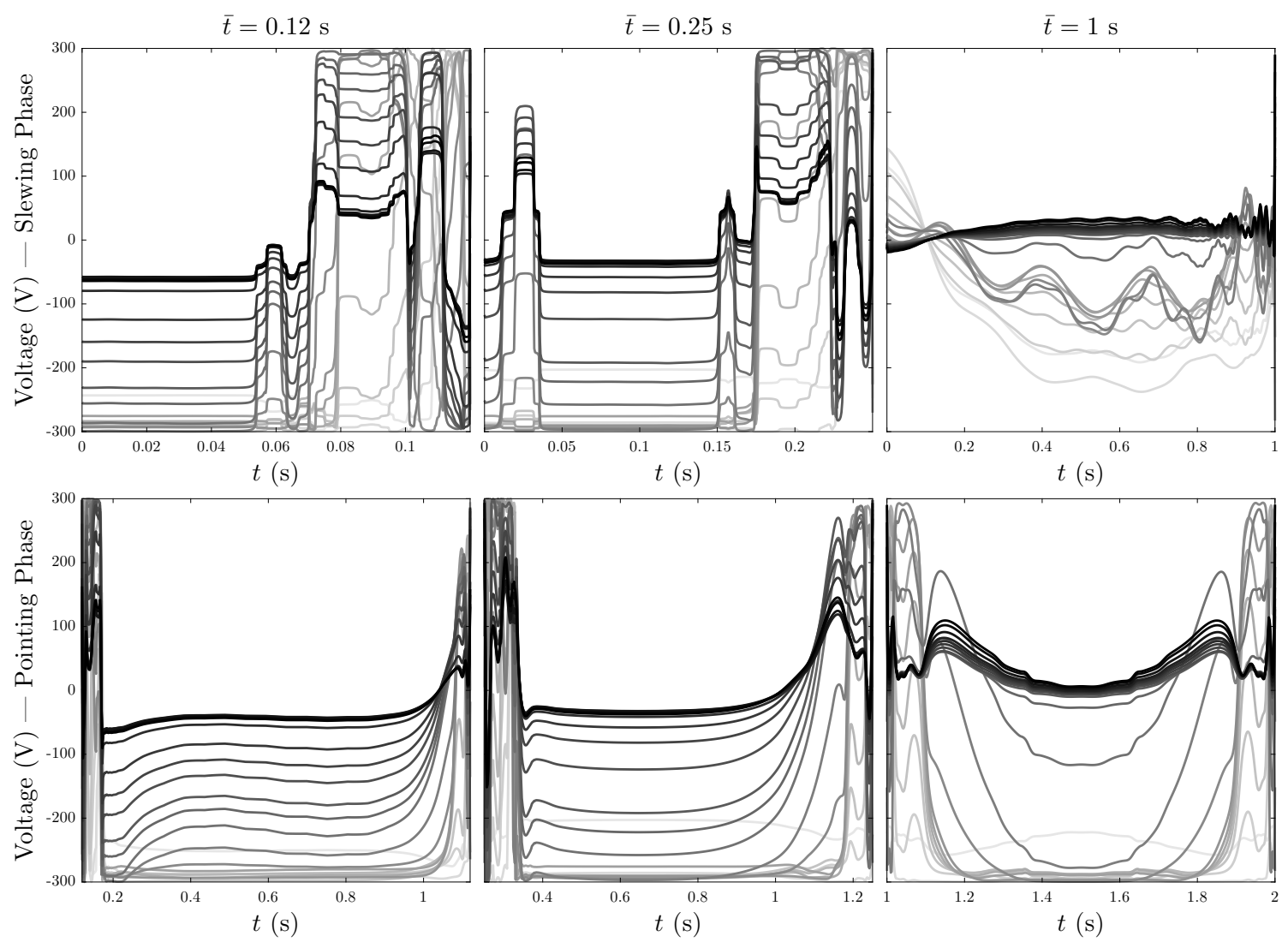

(a) Piecewise Linear Segments (PLS).
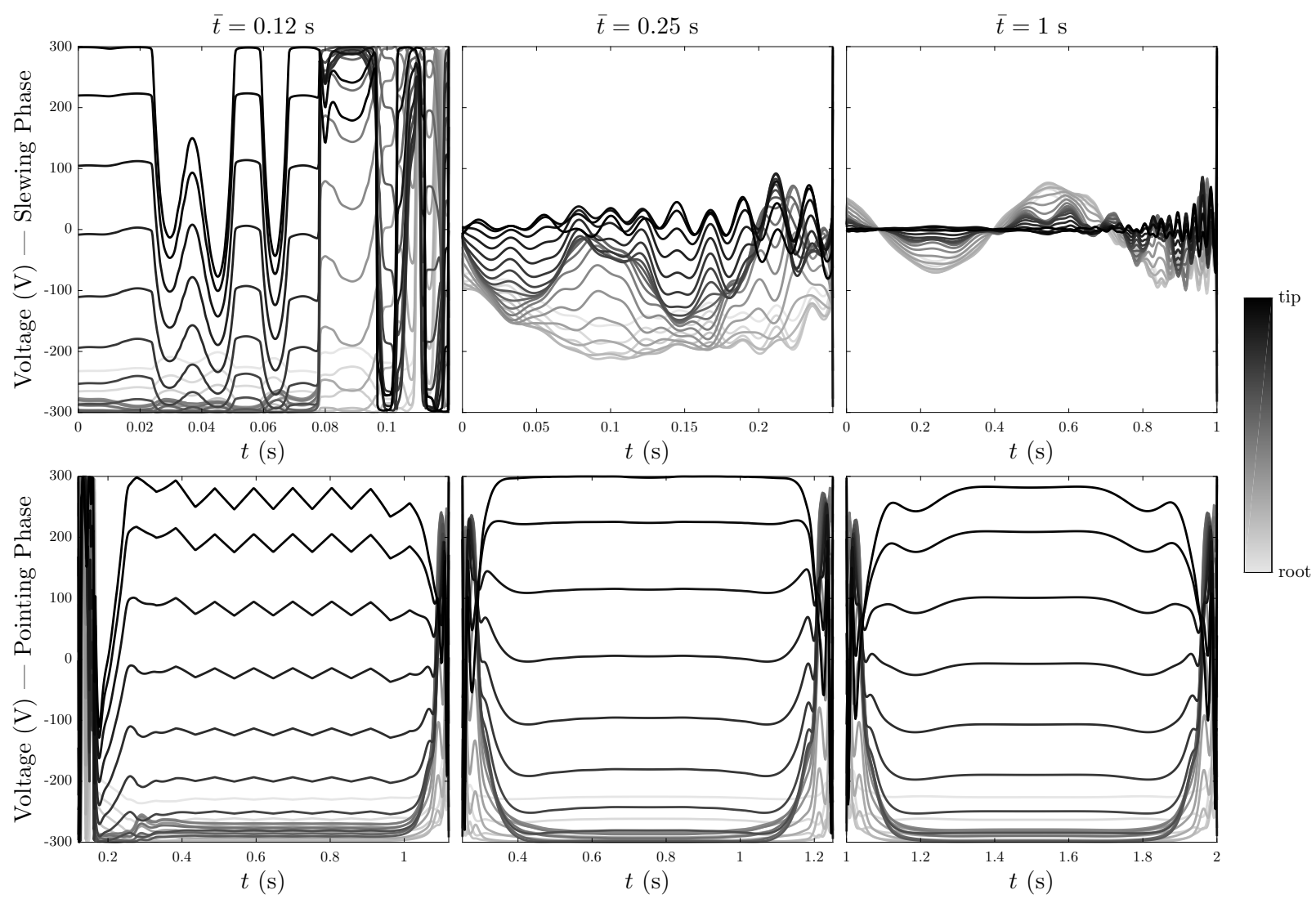

(b) Variable Length (VL).

Figure 8: Voltage history along the array for select values of $\bar{t}$. 29 of $\underline{43}$ 
to displace in the opposite direction (counter-clockwise) (see Fig. 1a). This is particularly evident in the VL case with slewing time of $1.0 \mathrm{sec}$, which shows the effect of CW and CCW array displacements on the bus angle trajectory in Figs. $\underline{9 \mathrm{~b}}$ and $\underline{7 \mathrm{~b}}$.

Figure $5 \mathrm{~b}$ shows the array physical design evolution with respect to the slewing time. For longer slewing times ( $\geq 0.5$ s for the PLS case and $\geq 0.25$ s for the VL case), the optimal array design maximizes the effective inertia ratio between the bus and the arrays subject to the given constraints. This array shape also corresponds to the maximum slew limits seen in the parametric study confirming the general result shown in Sec. IV.A. With this observation, we could define a cost proxy function for maximizing array inertia when we have long slewing times. $\stackrel{24}{ }$ With shorter slewing times, however, we see more complex designs that do not maximize inertia. Since the analysis using the PRBDM model did not take into account the control system, we need an alternate explanation for these optimal arrays designs. To this end, an integrated analysis is performed that considers the synergy between natural passive dynamics (natural modes of the array, no control) and active (controlled) dynamics.

\section{IV.C. Optimal Design Tradeoff for Array Structure}

The periods of the first natural modes for the array designs of the PLS and VL cases are shown in Fig. 10 (the NG case is not considered as it does not involve any modifications of the physical array design). The dashed line is a reference that indicates whether one quarter of the period of the first natural frequency, denoted $T_{1} / 4$, is longer (above) or shorter (below) than the given slewing time. We use the $T_{1} / 4$ line as a reference but the true relationship for this particular co-design formulation appears to be closer to $T_{1} / 4.2$, and likely varies slightly based on problem parameters. Consider now the array design that maximizes the array moment of inertia, subject to the given constraints, for a given problem variation with a particular value for $T_{1}$. If this particular value for $T_{1} / 4$ is shorter than the slewing time, the maximum inertia design will be optimal and the optimal controller will utilize primarily the passive dynamics of the first structural mode to achieve an optimal slewing maneuver (and use higher-order modes partially). If $T_{1} / 4$ is longer than the given slewing time, the 


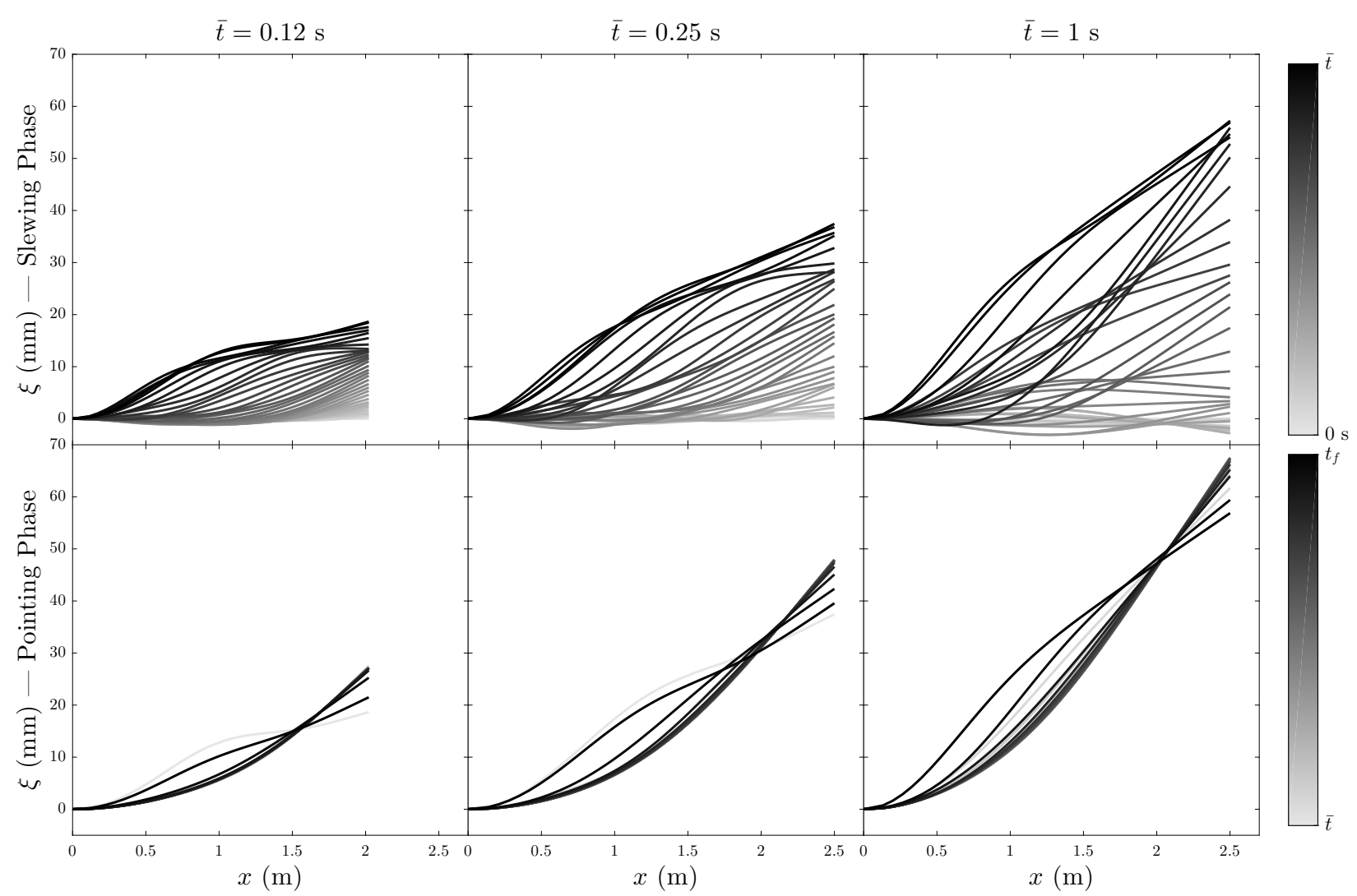

(a) Piecewise Linear Segments (PLS).

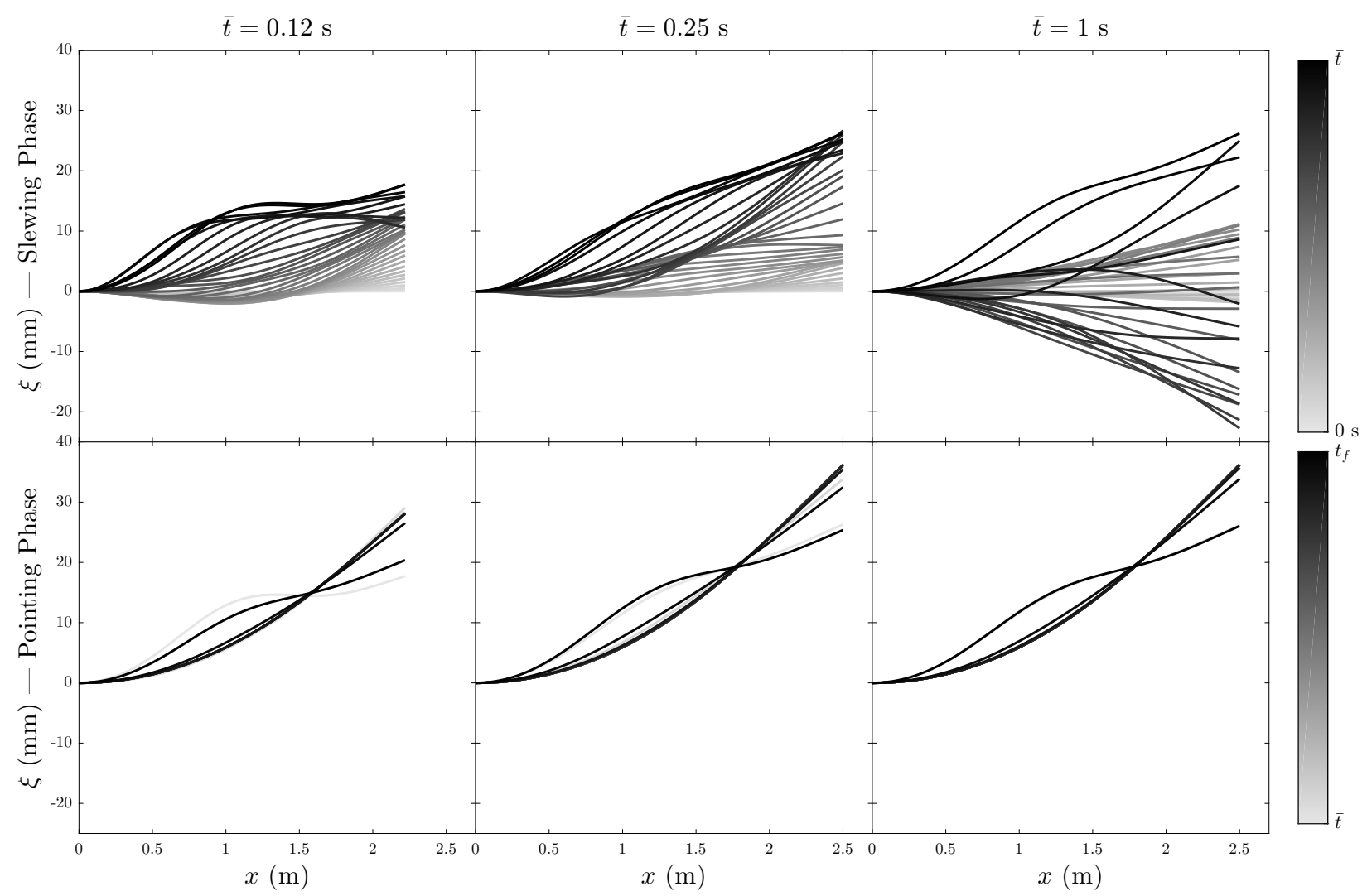

(b) Variable Length (VL).

Figure 9: Array deflection profiles for select values of $\bar{t}$.

31 of $\underline{43}$

American Institute of Aeronautics and Astronautics 
maximal inertia array design can only make partial use of the first mode dynamics in the slewing direction during the given slewing horizon. In other words, the array dynamics now need to be faster to work synergistically with the active controller when the slewing time is reduced, and the relationship between $\bar{t}$ and $T_{1}$ is approximately linear due to simple scaling of the problem based on the time horizon. Co-design studies are ideal for determining this tradeoff since allowing simultaneous structural and control design freedom provides access to higher performance levels through synergistic structural and control design tailoring without major assumptions, i.e., the parameters for the array structure and open-loop control design are distributed. These results also reveal that the proxy objective function of maximizing inertia is not accurate for faster slew times.

In the cases where the optimal tradeoff is active, the optimal control trajectories include 425 a bang-bang control near the root during the slewing phase, and the optimal array structure changes according to the given design freedom in Table $\underline{5}$. For the VL case, the only mechanism available for changing inertia and the first natural frequency is to adjust $\ell$, explaining the observed shorter array when $\bar{t}=0.12 \mathrm{~s}$ (see Fig. 5b). For the PLS case, the inertia and the first natural frequency are changed by redistributing the mass and/or reducing the array length to utilize more fully a combination of the array's elastic and inertial properties. Observe that the array mass is not reduced, i.e., the mass constraint in Eq. (19i) is active. In addition to using the first natural mode, results indicate that the optimal solutions with shorter $\bar{t}$ also tend to leverage the use of the second natural mode (refer to the increase in the coefficient of the second mode in Fig. 11). Tailoring of the structural design to best use the second mode is evident by the placement of a segment with local minimum thickness near the midpoint of the array length in Fig. 5b. The additional structural design freedom provided by the PLS formulation vs. the VL formulation demonstrates the ability of a co-design formulation with more plant design freedom to tailor the passive dynamics of the system to achieve better performance. $\underline{\underline{24}}$ 


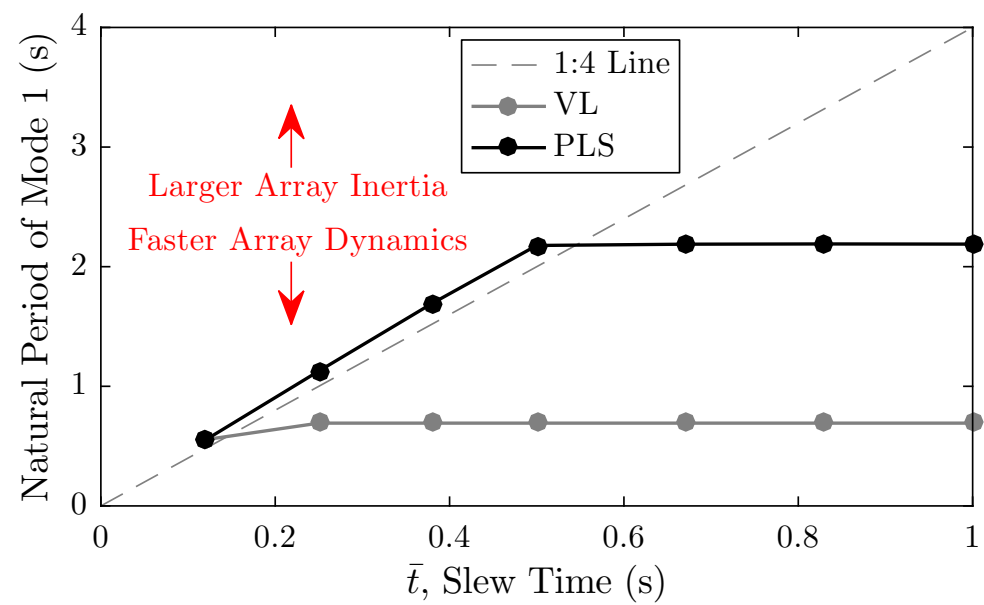

Figure 10: Comparison between the first natural period of the optimal array designs and the slewing phase duration.

\section{IV.D. Optimal Placement of Segmented Piezoelectric Actuators}

A continuously variable, spatially distributed voltage is not a physically realizable actuation strategy, but these optimal trajectories provide insights into performance limits, as well as how a physically realizable strain actuation system should be designed. Continuous voltage variation can be approximated using several piezoelectric segments as shown in Fig. $\underline{3 b}$, where a constant voltage $V_{i}(t)$ is applied to each segment. An analysis of optimal voltage trajectories for the PLS and VL cases was performed to provide insight into actuator placement. Figure 12 illustrates for each spatial position along the array 1) the maximum voltage amplitude across all time, 2) the mean voltage amplitude during slewing, and 3) the mean voltage amplitude during pointing. The maximum allowable voltage magnitude is $300 \mathrm{~V}$. For small $\bar{t}$, the actuators are nearly saturated during the slewing phase, and the voltage limit is reached at some point during the maneuver across most of the array. When the slewing times are longer, the lower average voltage magnitude during the slewing phase is due to the use of modal resonance.

We see that a physical implementation would benefit from placing piezoelectric segments over most of the array area with the exception of the tip. Since each piezoelectric segment can only be actuated with a voltage that is constant in space (not in time), a large number of individual segments translates into more degrees of freedom for the control, which in turn 

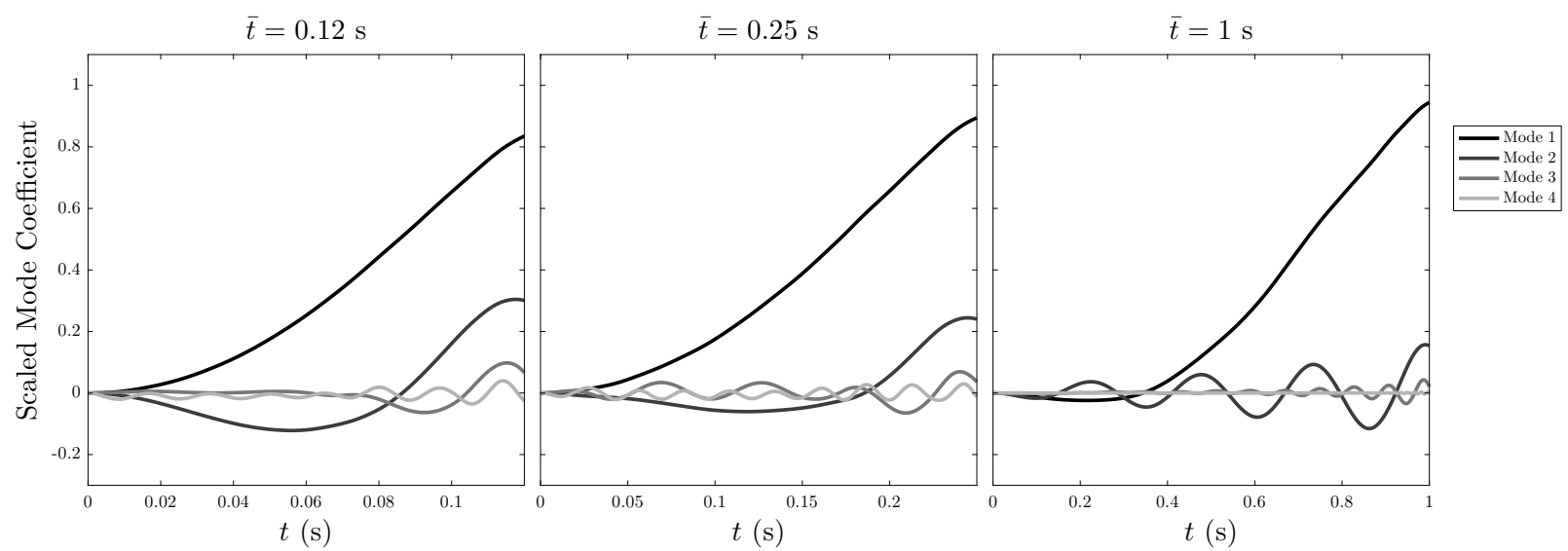

(a) Piecewise Linear Segments (PLS) design results.
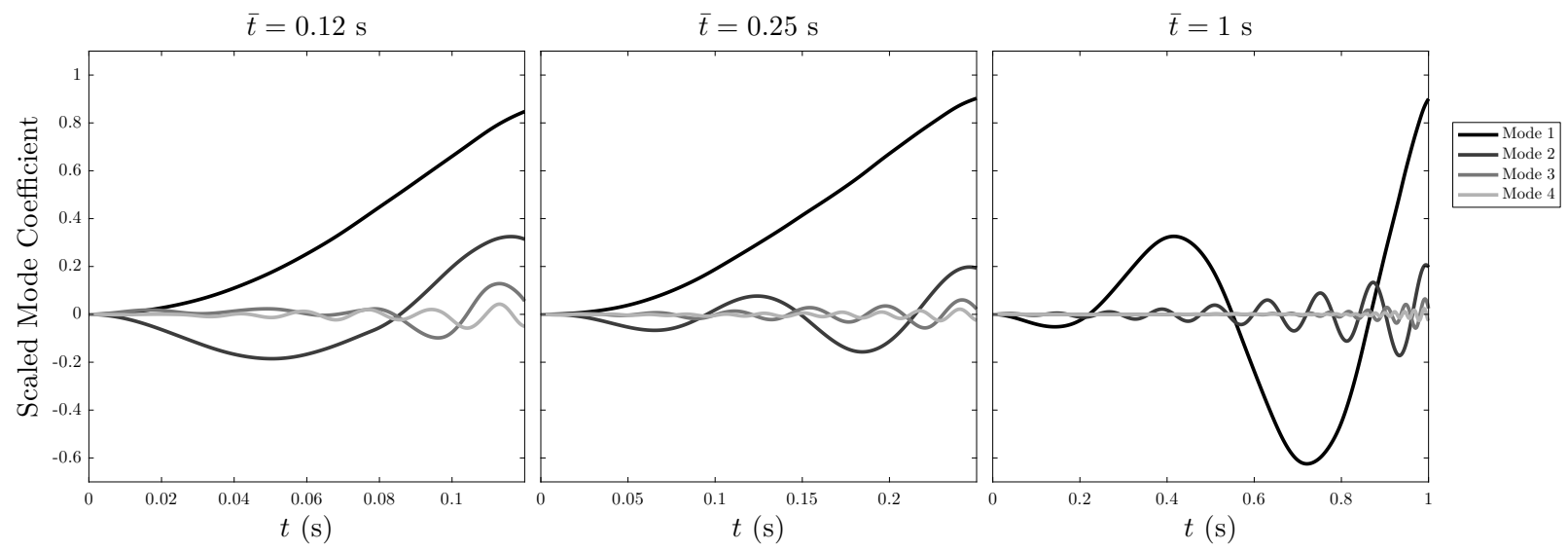

(b) Variable Length (VL) design results.

Figure 11: Scaled mode coefficient trajectories for select values of $\bar{t}$.

can allow higher performance. However, the voltage metrics suggest that at a minimum two piezoelectric segments should be placed at the two points of local maximum average voltage (which are located near the root and the length midpoint), to take advantage of the natural passive dynamics. These locations are near the critical points of the shapes of the first and second natural modes, which are the dominant modes during the slewing phase as shown in Fig. 11. The scaled mode coefficients in this figure indicate the relative contribution of each mode in their linearly combined effect on the array deflection. Future studies can include model actuation using individual piezoelectric segments with constant spatial voltage and limited length to determine their optimal placement location. 


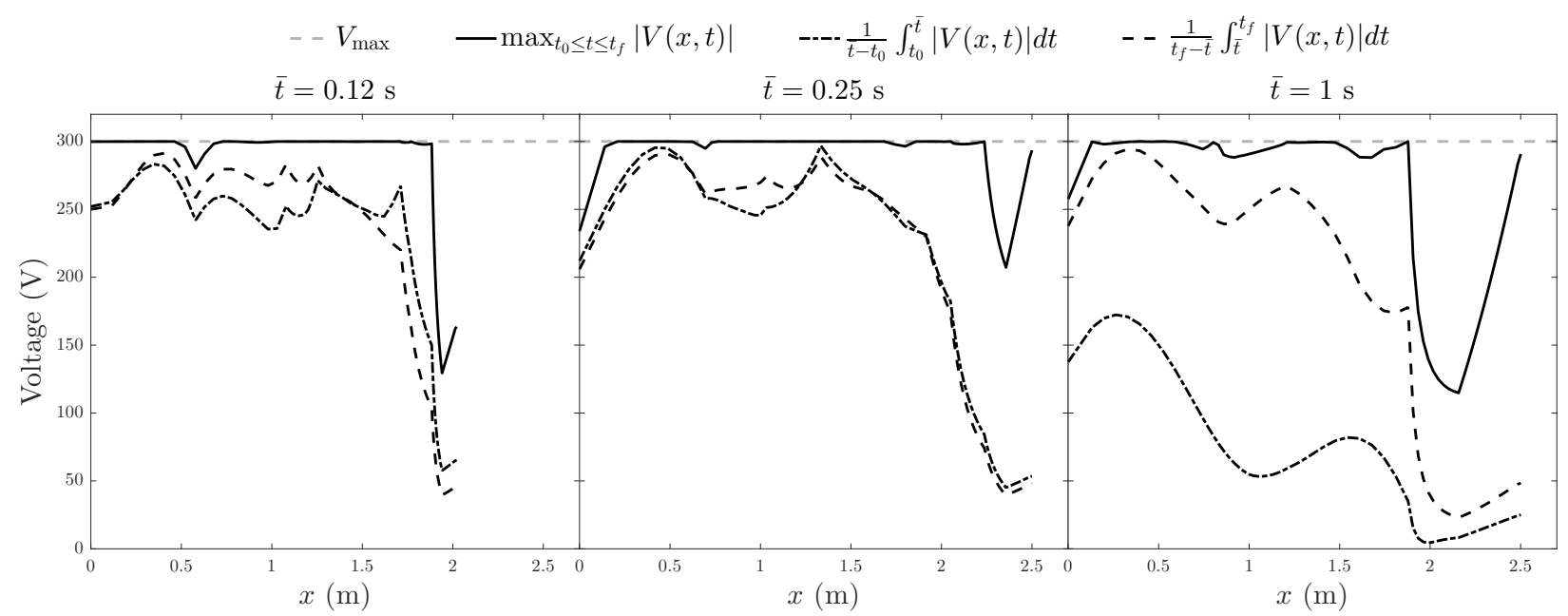

(a) Piecewise Linear Segments (PLS) design results.

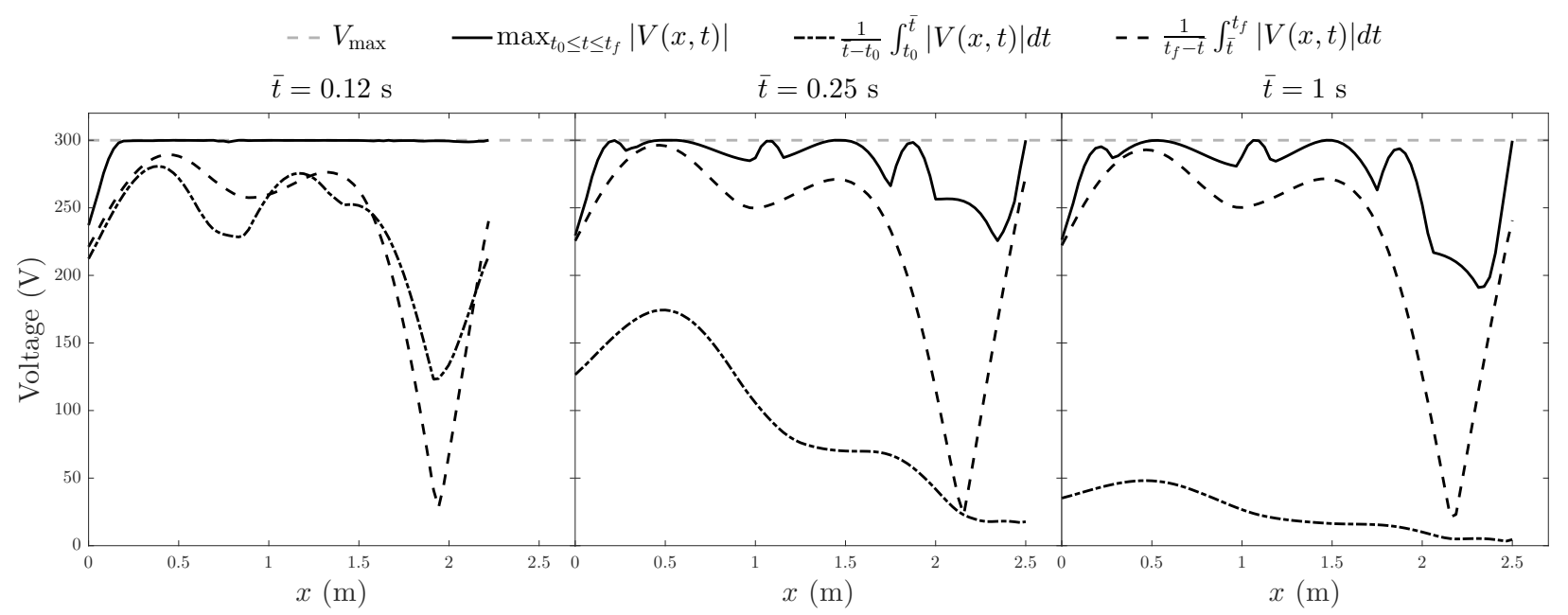

(b) Variable Length (VL) design results.

Figure 12: Voltage trajectory metrics for select values of $\bar{t}$.

\section{IV.E. Parametric Studies on Passive Damping and Jitter Disturbance}

Additional studies were conducted to determine the effect of passive damping and jitter disturbance on the performance of the SASA control system. As described in Eq. (7) and Table (2), the solar array model includes a passive damping coefficient $\mu$ with a nominal value of $10^{-4} \mathrm{~s}$. The equivalence between the coefficient $\mu$ and the damping ratio $\zeta$ of a second-order damped oscillator is shown in Eq. (23),

$$
2 \zeta_{i}=\mu \omega_{i}
$$




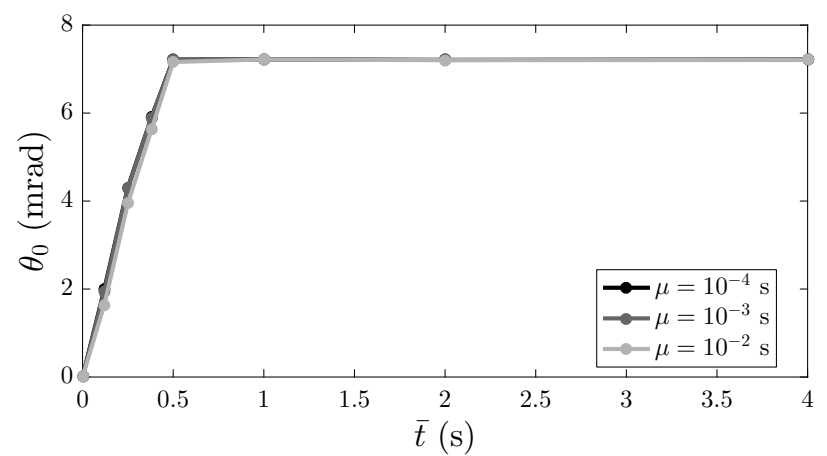

(a) Piecewise Linear Segments (PLS).

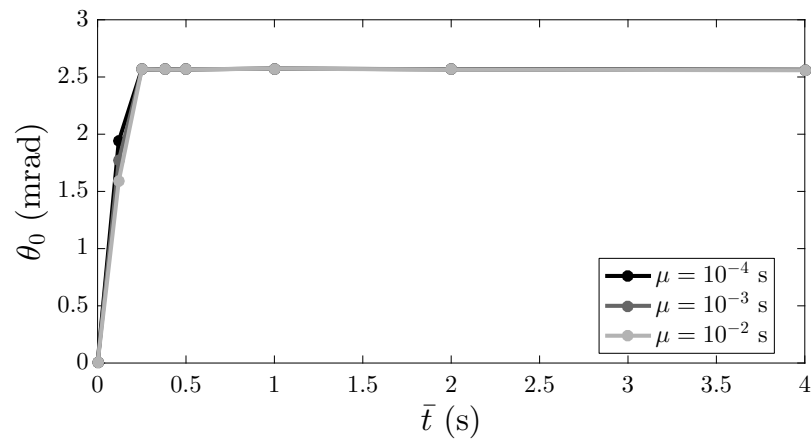

(b) Variable Length (VL).

Figure 13: Parametric studies of maximum slewing angle with respect to slewing time and passive damping.

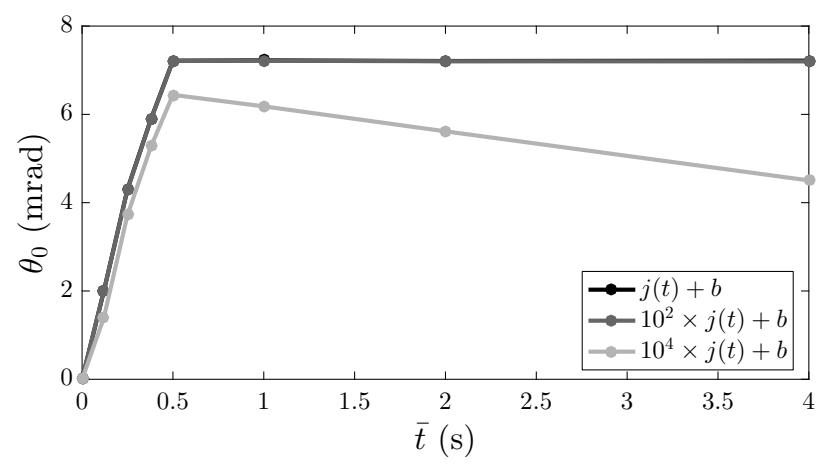

(a) Piecewise Linear Segments (PLS).

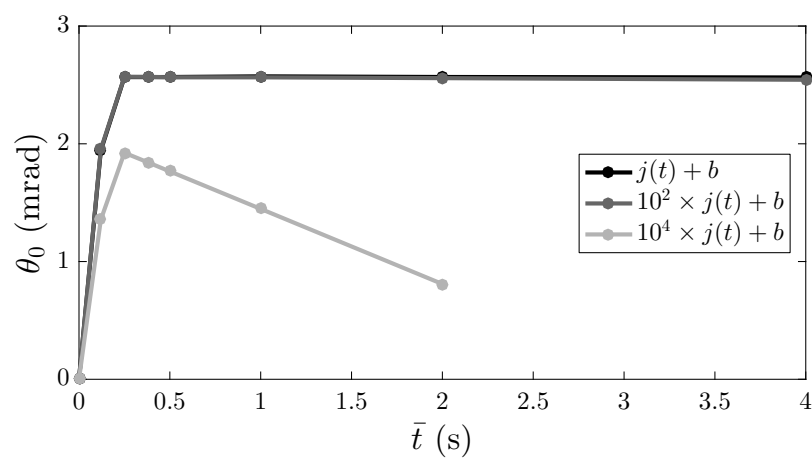

(b) Variable Length (VL).

Figure 14: Parametric studies of maximum slewing angle with respect to slewing time and jitter disturbance.

where $\omega$ is the natural frequency of the array and the subscript $i$ indicates the mode in consideration. Table (6) shows the respective damping ratios $\zeta_{1}$ for a given value of the coefficient $\mu$. Figure 13 shows the results of the parametric study with respect to passive damping for the PLS and VL cases using the optimal array designs that were determined for the given slewing times and the nominal damping coefficient. Although the effect of increasing the passive damping in the system performance is minimal, the largest differences occur when the slewing times are small. As expected, the effect of the array passive damping is larger when the array displacement rate is higher. The study shows that the optimal performance of the SASA system is robust with respect to changes in the array passive damping.

Equation (19b) shows that the bus is subject to a nominal disturbance, which includes a 
Table 6: Damping ratio $\zeta_{1}$ as a function of the coefficient $\mu$.

\begin{tabular}{rccccc}
\hline \hline \multicolumn{6}{c}{ Slewing time horizon (s) } \\
Variation & 0.12 & 0.25 & 0.38 & 0.5 & 1 \\
\hline PLS & $5.70 \mu$ & $2.79 \mu$ & $1.86 \mu$ & $1.44 \mu$ & $1.44 \mu$ \\
VL & $5.67 \mu$ & $4.54 \mu$ & $4.54 \mu$ & $4.54 \mu$ & $4.54 \mu$ \\
\hline \hline
\end{tabular}

jitter component $j(t)$ and a bias component $b$. The jitter component was varied parametri-

cally to investigate its influence on optimal design. Figure 14 shows the results of this study

for the PLS and VL cases using the optimal array designs that were determined for the given slewing times considering the nominal disturbance. For a disturbance factor of $10^{2} \times j(t)$, the jitter is completely managed and the maximum slewing angle is maintained similar to the nominal case, i.e., there is limited performance loss even with this high amplitude disturbance. Increasing the disturbance factor further to $10^{4} \times j(t)$ reduces significantly the performance of the system, and the maneuvers are infeasible for the VL case with a slewing time of $4 \mathrm{~s}$. PLS designs encounter feasibility problems when the disturbance factor is increased to $10^{5} \times j(t)$. The VL case is affected more by the disturbance because of its smaller inertia. This additional study demonstrates that the high bandwidth SASA control system is robust in canceling large jitter disturbances while maintaining high precision pointing.

\section{Conclusions}

In this work, we investigated the integrated structural and control system design of a strain-actuated solar array for spacecraft pointing control and jitter reduction. Slew maneuvers on the order of milli-radians or arc minutes have been achieved in simulations for a representative spacecraft system without increasing the total array mass or reducing the array planform area. A parametric study was conducted with different levels of design freedom and slewing times. Results show that separately designing the control system or the structural system alone cannot achieve the higher performance levels that are possible through the proposed combined design of the structural and control systems. Furthermore, adding degrees of freedom to the structural design - specifically, distributed geometric design-improved per- 
formance further by tailoring the passive dynamics of the array with the active controller.

Since the SASA system is based on internal actuation, the angular momentum of the busarray system must be conserved in the absence of material or joint damping. A desired bus rotation requires array deflection in the opposite direction. Results showed that in addition helped provide qualitative insights into the design and behavior of intelligent structures with distributed actuation.

\section{Acknowledgments}

Government sponsorship is acknowledged. The research was in part carried out at the Jet Propulsion Laboratory, California Institute of Technology under a contract with the National Aeronautics and Space Administration. The authors acknowledge help from Giri 
Subramanian and Kevin Lohan during the initial phases of the project.

\section{References}

${ }^{1}$ Arnon, S., Rotman, S., and Kopeika, N. S., "Optimum Transmitter Optics Aperture for Satellite Optical Communication," IEEE Transactions on Aerospace and Electronic Systems, Vol. 34, No. 2, Aug. 1998, pp. 590-596, doi: 10.1109/7.670339

${ }^{2}$ Ruiter, A. F. D., Damaren, C., and Forbes, J. R., Spacecraft Dynamics and Control: An Introduction, John Wiley \& Sons, 2013.

${ }^{3}$ Sirlin, S. W., "Vibration Isolation for Spacecraft Using the Piezoelectric Polymer PVF2," Journal of the Acoustical Society of America, Vol. 82, No. S13, Nov. 1987, doi: 10.1121/1.2024666

${ }^{4}$ Rao, S. S. and Sunar, M., "Piezoelectricity and Its Use in Disturbance Sensing and Control of Flexible Structures: A Survey," Applied Mechanics Reviews, Vol. 47, No. 4, April 1994, pp. 113-123, doi: $\underline{10.1115 / 1.3111074}$

${ }^{5}$ Cowen, R., "The Wheels Come Off Kepler," Nature, Vol. 497, No. 7450, 2013, pp. 417-418, doi: $10.1038 / 497417 \mathrm{a}$

${ }^{6}$ Coverstone-Carroll, V. L. and Wilkey, N. M., "Optimal Control of a Satellite-Robot System Using Direct Collocation with Non-linear Programming," Acta Astronautica, Vol. 36, No. 3, Aug. 1995, pp. 149162, doi: 10.1016/0094-5765(95)00096-I

Functions." IEEE Transactions on Control Systems Technology, Vol. 2, No. 3, Sept. 1994, pp. 245-254, doi: $\underline{10.1109 / 87.317981}$

${ }^{8}$ Manning, R. A., "Optimum Design of Intelligent Truss Structures," 32nd Structures, Structural Dynamics, and Materials Conference, Baltimore, MA, USA, April 1991, pp. 528-533, doi: $\underline{10.2514 / 6.1991-1158}$

${ }^{9}$ Smith, M. J., Grigoriadis, K. M., and Skelton, R. E., "Optimal Mix of Passive and Active Control in Structures," Journal of Guidance, Control, and Dynamics, Vol. 15, No. 4, July-Aug. 1992, pp. 912-919, doi: $10.2514 / 3.20924$

${ }^{10}$ Meirovitch, L., Dynamics and Control of Structures, John Wiley \& Sons, 1990.

39 of $\underline{43}$

American Institute of Aeronautics and Astronautics 
${ }^{11}$ Crawley, E. F. and Luis, J. D., "Use of Piezoelectric Actuators as Elements of Intelligent Structures," AIAA Journal, Vol. 25, No. 10, Oct. 1987, pp. 1373-1385, doi: 10.2514/3.9792

${ }^{12}$ Pan, J., Hansen, C. H., and Snyder, S. D., "A Study of the Response of a Simply Supported Beam to Excitation by a Piezoelectric Actuator," Journal of Intelligent Material Systems and Structures, Vol. 3, No. 1, Jan. 1992, pp. 3-16, doi: 10.1177/1045389X9200300101

${ }^{13}$ Hubbard Jr., J. E. and Burke, S. E., "Distributed Transducer Design for Intelligent Structural Components," Intelligent Structural Systems, edited by H. S. Tzou and G. L. Anderson, Vol. 13, Springer, 1992, pp. 305-324, doi: 10.1007/978-94-017-1903-2_8

${ }^{14}$ Hwang, W. and Park, H. C., "Finite Element Modeling of Piezoelectric Sensors and Actuators," AIAA Journal, Vol. 31, No. 5, May 1993, pp. 930-937, doi: 10.2514/3.11707

${ }^{15}$ Alvarez-Salazar, O. S. and Iliff, K., "Destabilizing Effects of Rate Feedback on Strain Actuated Beams," Journal of Sound and Vibration, Vol. 221, No. 2, March 1999, pp. 289-307, doi: 10.1006/jsvi.1998.2010

${ }^{16}$ Bailey, T. and Hubbard Jr., J. E., "Distributed Piezoelectric-Polymer Active Vibration Control of a Cantilever Beam," Journal of Guidance, Control, and Dynamics, Vol. 8, No. 5, Sept. 1985, pp. 605-611, doi: $10.2514 / 3.20029$

${ }^{17}$ Dimitriadis, E. K., Fuller, C. R., and Rogers, C. A., "Piezoelectric Actuators for Distributed Vibration Excitation of Thin Plates," Journal of Vibration and Acoustics, Vol. 113, No. 1, Jan. 1991, pp. 100-107, doi: $10.1115 / 1.2930143$

${ }^{18}$ Pinkerton, J. L., McGowan, A.-A. R., Moses, R. W., Scott, R. C., , and Heeg, J., "Controlled Aeroelastic Response and Airfoil Shaping Using Adaptive Materials and Integrated Systems," Symposium on Smart Structures and Materials, San Diego, CA, USA, Feb. 1996, pp. 166-177.

${ }^{19}$ Fowler, R. M., Investigation of Compliant Space Mechanisms with Application to the Design of a Large-Displacement Monolithic Compliant Rotational Hinge, M.S. Thesis, Brigham Young University, Aug. 2012 .

${ }^{20}$ Balas, M. J., "Active Control of Flexible Systems," Journal of Optimization Theory and Applications, Vol. 25, No. 3, July 1978, pp. 415-436, doi: 10.1007/BF00932903

${ }^{21}$ Junkins, J. L. and Rew, D. W., "Unified Optimization of Structures and Controllers," Large Space Structures: Dynamics and Control, edited by S. N. Atluri and A. K. Amos, Springer Series in Computational Mechanics, Springer, 1988, pp. 323-353, doi: 10.1007/978-3-642-83376-2_14

${ }^{22}$ Allison, J. T., Guo, T., and Han, Z., "Co-Design of an Active Suspension Using Simultane585 ous Dynamic Optimization," Journal of Mechanical Design, Vol. 136, No. 8, Aug. 2014, pp. 081003, doi: $10.1115 / 1.4027335$

40 of $\underline{43}$ 
${ }^{23}$ Allison, J. T. and Herber, D. R., "Multidisciplinary Design Optimization of Dynamic Engineering Systems," AIAA Journal, Vol. 52, No. 4, April 2014, pp. 691-710, doi: 10.2514/1.J052182

${ }^{24}$ Allison, J. T., "Plant-Limited Co-Design of an Energy-Efficient Counterbalanced Robotic Manipula-

tor," ASME Journal of Mechanical Design, Vol. 135, No. 10, 2013, pp. 101003, doi: 10.1115/1.4024978

${ }^{25}$ Salama, M., Garba, J., and Demsetz, L., "Simultaneous Optimization of Controlled Structures," Computational Mechanics, Vol. 3, No. 4, July 1988, pp. 275-282, doi: 10.1007/BF00368961

${ }^{26}$ Wu, Z., Harne, R. L., and Wang, K.-W., "Energy Harvester Synthesis Via Coupled Linear-Bistable System with Multistable Dynamics," Journal of Applied Mechanics, Vol. 81, No. 6, Feb. 2014, pp. 061005, doi: $10.1115 / 1.4026555$

${ }^{27}$ Grigoriadis, K. M., Zhu, G., and Skelton, R. E., "Optimal Redesign of Linear Systems," Journal of Dynamic Systems, Measurement, and Control, Vol. 118, No. 3, Sept. 1993, pp. 598-605, doi: $\underline{10.1115 / 1.2801186}$

${ }^{28}$ Allison, J. T., Kokkolaras, M., and Papalambros, P. Y., "On Selecting Single-Level Formulations for Complex System Design Optimization," Journal of Mechanical Design, Vol. 129, No. 9, Sept. 2006, pp. 898906, doi: $\underline{10.1115 / 1.2747632}$

${ }^{29}$ Herber, D. R., Dynamic System Design Optimization of Wave Energy Converters Utilizing Direct Transcription, M.S. Thesis, University of Illinois at Urbana-Champaign, Urbana, IL, USA, May 2014.

${ }^{30}$ Fanson, J. L. and Caughey, T. K., "Positive Position Feedback Control for Large Space Structures," AIAA Journal, Vol. 28, No. 4, April 1990, pp. 717-724, doi: 10.2514/3.10451

${ }^{31} \mathrm{Li}$, W. P. and Huang, H., "Integrated Optimization of Actuator Placement and Vibration Control for Piezoelectric Adaptive Trusses," Journal of Sound and Vibration, Vol. 332, No. 1, Jan. 2013, pp. 17-32, doi: $10.1016 /$ j.jsv.2012.08.005

${ }^{32}$ Grandhi, R. V., "Structural and Control Optimization of Space Structures," Computers 8 Structures, Vol. 31, No. 2, 1989, pp. 139-150, doi: 10.1016/0045-7949(89)90222-8

${ }^{33}$ Belvin, W. K. and Park, K. C., "Structural Tailoring and Feedback Control Synthesis: An Interdisciplinary Approach," Journal of Guidance, Control, and Dynamics, Vol. 13, No. 3, May 1990, pp. 424-429, doi: $10.2514 / 3.25354$

${ }^{34}$ Herber, D. R., McDonald, J. W., Alvarez-Salazar, O. S., Krishnan, G., and Allison, J. T., "Reducing Spacecraft Jitter During Satellite Reorientation Maneuvers via Solar Array Dynamics," AIAA/ISSMO Multidisciplinary Analysis and Optimization Conference, No. AIAA 2014-3278, Atlanta, GA, USA, June 2014, doi: $10.2514 / 6.2014-3278$

${ }^{35}$ Alvarez-Salazar, O. S., Aldrich, J. B., Filipe, N., Allison, J. T., and Chung, S.-J., "Strain Actuated 41 of $\underline{43}$ 
Solar Arrays for Precision Pointing of Spacecraft," AAS Guidance, Navigation, and Control Conference, No.

AAS 16-137, Breckenridge, CO, USA, Feb. 2016.

${ }^{36}$ Yu, Y.-Q., Howell, L. L., Lusk, C., Yue, Y., and He, M.-G., "Dynamic Modeling of Compliant Mechanisms Based on the Pseudo-Rigid-Body Model," Journal of Mechanical Design, Vol. 127, No. 4, Aug. 2005, pp. 760-765, doi: $10.1115 / 1.1900750$

${ }^{37}$ Ben-Tal, A. and Bar-Yoseph, P. Z., "Space-Time Spectral Element Method for Optimal Slewing of a Flexible Beam," International Journal for Numerical Methods in Engineering, Vol. 39, No. 18, Sept. 1996, pp. 3101-3121, doi: 10.1002/(SICI)1097-0207(19960930)39:18<3101::AID-NME987>3.0.CO;2-O

${ }^{38}$ Chilan, C. M., Herber, D. R., Nakka, Y. K., Chung, S.-J., Allison, J. T., Aldrich, J. B., and AlvarezSalazar, O. S., "Co-Design of Strain-Actuated Solar Arrays for Precision Pointing and Jitter Reduction," AIAA/ASCE/AHS/ASC Structures, Structural Dynamics, and Materials Conference, No. AIAA 2016-0162, San Diego, CA, USA, Jan. 2016, doi: 10.2514/6.2016-0162

${ }^{39}$ Bisplinghoff, R. L., Ashley, H., and Halfman, R. L., Aeroelasticity, Addison-Wesley, 1995.

${ }^{40}$ Junkins, J. L. and Kim, Y., Introduction to Dynamics and Control of Flexible Structures, AIAA, 1st ed., 1993, doi: 10.2514/4.862076

${ }^{41}$ Paranjape, A. A., Guan, J., Chung, S.-J., and Krstic, M., "PDE Boundary Control for Flexible Articulated Wings on a Robotic Aircraft," IEEE Transactions on Robotics, Vol. 29, No. 3, June 2013, pp. 625-640, doi: 10.1109/TRO.2013.2240711

${ }^{42}$ Deshmukh, A. P., Herber, D. R., and Allison, J. T., "Bridging the Gap between OpenLoop and Closed-Loop Control in Co-Design: A Framework for Complete Optimal Plant and Control Architecture Design," American Control Conference, Chicago, IL, USA, July 2015, pp. 4916-4922, doi: 10.1109/ACC.2015.7172104

${ }^{43}$ Paranjape, A. A., Chung, S.-J., Hilton, H. H., and Chakravarthy, A., "Dynamics and Performance of Tailless Micro Aerial Vehicle with Flexible Articulated Wings," AIAA Journal, Vol. 50, No. 5, May 2012, pp. 1177-1188, doi: 10.2514/1.J051447

${ }^{44}$ Nakka, Y. K., Nonlinear Attitude Control of Spacecraft With Strain-Actuated Solar Arrays, M.S. Thesis, University of Illinois at Urbana-Champaign, Urbana, IL, USA, Aug. 2016.

${ }^{45}$ Moheimani, S. O. M. and Fleming, A. J., Piezoelectric Transducers for Vibration Control and Damping, Springer, 1st ed., 2006, doi: 10.1007/1-84628-332-9

${ }^{46}$ Lindmayer, J. and C.Y., W., "Development of a High Efficiency Thin Silicon Solar Cell," Tech. Rep. SX/105/F, Solarex Corporation, Rockville, MD, USA, Sept. 1977.

${ }^{47}$ Lee, J., Wu, J., Shi, M., Yoon, J., Park, S.-I., Li, M., Liu, Z., Huang, Y., and Rogers, J. A., "Stretchable

42 of $\underline{43}$ 
GaAS Photovoltaics With Designs That Enable High Areal Coverage," Advanced Materials, Vol. 23, No. 8, Feb. 2011, pp. 986-991, doi: 10.1002/adma.201003961

${ }^{48}$ Qiu, L., He, S., Yang, J., Deng, J., and Peng, H., "Fiber-Shaped Perovskite Solar Cells With High Power Conversion Efficiency," Small, Vol. 12, No. 18, May 2016, pp. 2419-2424,

doi: $10.1002 /$ smll.201600326

${ }^{49}$ Fathy, H. K., Reyer, J. A., and Papalambros, P. Y., "On the Coupling Between the Plant and Controller Optimization Problems," American Control Conference, Arlington, VA, USA, June 2001, pp. 1864-1869, doi: 10.1109/ACC.2001.946008

${ }^{50}$ Enright, P. J. and Conway, B. A., "Discrete Approximations to Optimal Trajectories Using Direct Transcription and Nonlinear Programming," Journal of Guidance, Control, and Dynamics, Vol. 15, No. 4, July-Aug. 1992, pp. 994-1002, doi: 10.2514/3.20934

${ }^{51}$ Betts, J. T., Practical Methods for Optimal Control and Estimation Using Nonlinear Programming, SIAM, 2nd ed., 2010, doi: 10.1137/1.9780898718577

${ }^{52}$ Biegler, L. T., Nonlinear Programming: Concepts, Algorithms, and Applications to Chemical Processes, SIAM, 2010, doi: $10.1137 / 1.9780898719383$

${ }^{53}$ Chilan, C. M. and Conway, B. A., "Automated Design of Multiphase Space Missions Using Hybrid Optimal Control," Journal of Guidance, Control, and Dynamics, Vol. 36, No. 5, Sept. 2013, pp. 1410-1424, doi: $\underline{10.2514 / 1.58766}$

${ }^{54}$ Morgan, D., Chung, S.-J., and Hadaegh, F. Y., "Model Predictive Control of Swarms of Spacecraft Using Sequential Convex Programming," Journal of Guidance, Control, and Dynamics, Vol. 37, No. 6, 2014, pp. 1725-1740, doi: 10.2514/1.G000218

43 of $\underline{43}$ 